\title{
Ferromagnetism in (III,Mn)V Semiconductors
}

Jürgen König, John Schliemann, Tomáš Jungwirth, and Allan H. MacDonald

\section{Introduction}

Ferromagnetism occurs when Mn is randomly substituted for more than about 2 percent of the cations of several III-V compound semiconductors. Although only a few host materials have been explored at present, this property is likely shared by most III-V semiconductors. In this Chapter we will discuss some of the theoretical pictures that are being developed to explain the magnetic and transport properties of these materials. Our development will be based on a phenomenological model that has been used with great success to explain the sensitivity of bulk and layered (II,Mn)VI semiconductor optical properties to external magnetic fields. (Ferromagnetism does not occur for Mn in undoped II-VI hosts.) The low energy degrees of freedom in this model are holes in the semiconductor valence band and one $S=5 / 2$ local moment for each $\mathrm{Mn}$ ion.

Interest in these ferromagnets was heightened by the demonstration several years ago that ferromagnetic transition temperatures [i] in excess of $100 \mathrm{~K}$ can be achieved in (Ga,Mn)As. It has been further heightened recently both by the demonstration of long spin-coherence times in semiconductors [2] and by the dramatic and rapid development of new information storage technology based on magnetotransport effects in ferromagnetic metals [3]. It seems clear that semiconductors have many potential advantages over metals for devices based on the magnetotransport effects that occur in itinerant electron ferromagnets, principally because they present a wider canvas for their creative manipulation by some combination of impurities, gates and optical excitation. It is likely that important applications for these materials will be found only if ferromagnetism at room temperature can be achieved. The very recent discovery [ [ $1000 \mathrm{~K}$ in $(\mathrm{Ga}, \mathrm{Mn}) \mathrm{N}$ has fueled hopes that these materials will indeed have technological impact. Our focus here, however, is on the physics of these ferromagnets; we therefore concentrate mainly on the properties of (Ga,Mn)As and (In,Mn)As which have been studied most extensively [5].

It is generally accepted that Mn acts as an acceptor when it substitutes for a cation in a III-V semiconductor lattice, leaving a $\mathrm{Mn}^{2+}$ ion which has a half-filled d-shell with angular momentum $L=0$ and spin $S=5 / 2$. It is also generally accepted that ferromagnetism occurs in these materials because of 
interactions between $\mathrm{Mn}$ local moments that are mediated by holes in the semiconductor valence band. There is, therefore, a lot of similarity between the ferromagnetism of these materials and that of lanthanides and actinides and their compounds in which f-electron moments are coupled by d-band itinerant electrons. There are also similarities between these materials and the manganite compounds that have been extensively studied [6] in recent years in part because of the large increase in resistance that occurs when $T$ exceeds the Curie temperature, the so-called colossal magnetoresistance effect. In (III,Mn)V ferromagnets, however, the local moments appear on only a small fraction of the atomic sites arranged randomly. In addition the itinerant electron density is also low, even lower than the Mn local moment density. As we explain later this property is likely important in selecting ferromagnetic over glassy magnetic order. The participation of itinerant electrons in the ferromagnetism of these diluted magnetic semiconductors (DMS) adds to their richness, leading in particular to electronic transport properties that are very sensitive to the magnetic state of the material. The physics of ferromagnetic semiconductors is in a sense intermediate between that of rare earth magnets and that of manganites in that the spin-splitting of itinerant electron bands due to their exchange coupling with local moments is comparable to their Fermi energies, rather than being much smaller than band Fermi energies as in the rare earth case or larger as in the manganite case.

The Chapter is organized as follows. In Section 2 we briefly summarize the main experimental properties of (III,Mn)V ferromagnets. Several different but related approaches that have been explored in an effort to gain insight into these materials are outlined in Section 3. The remaining sections of this review Chapter deal with the development of the semi-phenomenological model we favor in which the low energy degrees of freedom are exchange-coupled valence-band holes and $S=5 / 2 \mathrm{Mn}$ local moments that carry a negative charge. The simplest version of this model is one in which the randomly distributed $\mathrm{Mn}$ ions are replaced by a uniform continuum, thus completely neglecting disorder. In Section 1 we discuss physical predictions based on a mean-field treatment of this disorder-free model and demonstrate that it successfully describes a number of non-trivial properties of ( $\mathrm{Ga}, \mathrm{Mn}) \mathrm{As}$ and (In,Mn)As ferromagnets, including their anomalous Hall conductivities. In Section 5 we discuss collective excitations of these ferromagnets within the disorder-free model, demonstrating that the simple mean-field-theory is reasonably reliable for typical parameters of current samples but must fail at large carrier densities and also in the limit of very strong exchange coupling. In Section 6 we discuss the results of Monte Carlo calculations that describe the effect of collective fluctuations of Mn moment orientations. The method can deal with some of the complications and additional physics, including the possibility of non-collinear ground states, that enters when disorder is added to the theoretical model. A brief summary is given in Section 7 . 
In Sections 1 . 5 , and 6 we have been able to present only a small fraction of theoretical data obtained using our approach. An extensive survey of predicted physical properties of DMS's will be available at http:// unix12.fzu.cz/ ms/index. php web-pages, launched by the authors of this Chapter in collaboration with Jan Kučera, Byounghak Lee, and Jairo Sinova.

\section{Properties of (III,Mn)V Ferromagnets}

In this section we discuss some important properties of (III,Mn)V ferromagnets that have been established by current experiments. Thorough recent reviews of the properties of these materials have been prepared by Dietl, Matsukura, and Ohno [7,8]. Our objective in this section is to summarize the observations that are most important in constraining theoretical descriptions. There is at present considerable activity related to the growth and characterization of these materials. We expect rapid progress to be achieved in the near future in exploring the range of possible behaviors and relating them more precisely to molecular-beam-epitaxy growth and post-growth annealing protocols. We list below a number of properties that appear to be safely established. A theoretical picture that is able to explain most of these properties is outlined in the following sections.

- Electron paramagnetic resonance and optical experiments [9, 10] demonstrate that $S=5 / 2$ local moments occur for dilute concentrations of $\mathrm{Mn}$ in GaAs. These experiments demonstrate that the Mn local moment model is correct.

- The Mn-induced states near the Fermi energy play a key role in the origin of ferromagnetism and in the magnetotransport properties of (III,Mn) V DMS's. According to photoemission studies [11, 12, 13], those states have As $4 p$ character, i.e., can be associated with the host semiconductor valence band states. The angle-resolved photoemission experiment also showed a negligible shift in the heavy- and light-hole bands with Mn concentration $x \leq 7 \%$.

- Ferromagnetism is not observed for Mn concentrations smaller than $\sim$ 0.01 [5]. This property demonstrates that ferromagnetism does not occur when all valence-band holes are trapped on individual Mn ions or on other defects. Antisite defects, for example, are common in semiconductor samples grown by low-temperature molecular beam epitaxy (MBE). For very dilute $\mathrm{Mn}$ concentrations, electron spin resonance experiments demonstrate that most holes are trapped not at the Mn acceptors, but at other defects.

- The ground-state magnetization, $M(T=0)$, per Mn ion can exceed $4 \mu_{B}$ for larger values of $x$ [5]. Since the magnetization contribution from antiferromagnetically coupled valence band holes tends to partially compensate the Mn local moment magnetization, ferromagnets with these large 
values of $M(T=0)$ likely have ground states with (nearly) fully aligned Mn local moments.

- To date the largest ferromagnetic transition temperatures in (Ga,Mn)As occur for $x \sim 5 \%$. The current record is $T_{c} \sim 110 K$ [1]. The drop in critical temperatures at higher $x$ values may be related to Mn clustering or may have a more fundamental origin. There is a correlation between large values of $M(T=0)$ and high $T_{c}$ 's.

- (III,Mn)V thin film ferromagnets grown under compressive strain have their magnetic easy axis in the plane, while ferromagnets grown under tensile strain have their magnetic easy axis in the growth direction [5]. External magnetic fields $\sim 100 \mathrm{mT}$ are sufficient to align the magnetization along the hard axis 14,7 . These properties can be explained by well understood strain effects in the spin-orbit coupled valence bands and demonstrate that the macroscopic properties of these ferromagnets are sensitive to details of the valence band electronic structure.

- The ferromagnetic critical temperature and the temperature dependence of the magnetization are altered by post-growth annealing and are sensitive to the details of the annealing protocol [5].

- These ferromagnets have large anomalous Hall resistivities [5], demonstrating that the itinerant valence bands are full participants in the magnetism. The large anomalous Hall resistivities reflect the strong spin-orbit coupling that is present at the top of the valence band in zincblende semiconductors.

- The semiconductor valence bands are spin-split in the ferromagnetic state. These semiconductor ferromagnets exhibit strong magnetoresistance effects, like tunnel magnetoresistance [15], that are characteristic of itinerant electron ferromagnets, again demonstrating that the itinerant electrons are full participants in the magnetism.

\section{Theoretical Approaches}

Ferromagnetism is a collective effect due to interactions between electrons. If it were possible to do so, we would explain its microscopic origins in a particular class of materials by solving the many-electron Schrödinger equation directly, given the position of all the nuclei. Fortunately this uninteresting direct approach is impossible, now and forever more, because of the macroscopic number of interacting electronic degrees of freedom. Instead we must resort to some combination of approximation and phenomenological modeling, settling on the correct approach only after careful comparison with experiment. This is the art of condensed matter science; an intricate tango between theory and experiment leading to a conclusion that cannot be anticipated while the dance is in progress. In some cases, fractional quantum Hall systems and possibly cuprate superconductors for example, the low-energy degrees of freedom in terms of which observable physical properties are best 
described are not simply related to the bare electrons which appear in the many-electron Hamiltonian. Often, however, the low-energy degrees of freedom are more obvious.

A practical approach to many-electron physics that is often successful is spin-density-functional (SDF) theory, in which many-body effects appear in exchange-correlation potential contributions to effective independent-particle Hamiltonians. SDF theory has the advantage that it is a first principles approach without any phenomenological parameters. Among its many achievements is a generally satisfactory description of itinerant electron ferromagnetism in transition metals. SDF theory has been applied [16 to (III,Mn)V ferromagnetism by Sanvito et al. and by van Schilfgaarde and Mryasov. To date these calculations have been performed using the local density approximation (LDA) of SDF theory, an approximation that is not reliable when local moments are formed, i.e., when strong correlations suppress fluctuations in the number of electrons in the d-shell or the f-shell of a particular atom. LDA-SDF theory supercell [16] calculations predict that majority spin d-electrons of a Mn atom substituted on a cation site of GaAs lie at the Fermi energy, rather than lying well below the Fermi energy as they would if the half-filled d-shell formed a $S=5 / 2$ local moment. Similar results have been obtained in coherent potential approximation band-structure calculations 17] for $(\mathrm{In}, \mathrm{Mn}) \mathrm{As}$, and are clearly in disagreement with experiment in both cases. In local moment systems, it is necessary to account for the increase in instantaneous cite energy when the occupancy of a localized orbital is increased. The LDA+U method has been developed to mitigate this deficiency of SDF theory and has recently 18 been applied to $(\mathrm{III}, \mathrm{Mn}) \mathrm{V}$ ferromagnets and finds a dominant As $4 p$ orbital weight at the Fermi energy, consistent with the photoemisson experiment.

It appears likely that a lot of detailed information on the electronic properties of (III,Mn)V ferromagnetic semiconductors can be reliably obtained from LDA+U SDF calculations and we expect this approach will play an important role in the modeling of these materials in the future. Our work, however, follows a semi-phenomenological strategy, starting from a model in which the local-moment character of the $\mathrm{Mn}$ d-orbitals is asserted rather than derived. These models are adapted from ones used 19,20 to describe the optical properties of (II,Mn)VI semiconductors. The low-energy degrees of freedom in the kinetic-exchange model [21,22] we employ are $S=5 / 2$ local moments representing half-filled Mn d-shells, and holes in the Mn valence band (see Fig. 1). Since $\mathrm{Mn}^{2+}$ ions should act as acceptors when substituted on the cation sites of III-V semiconductors, we would expect one hole per Mn if no other charged defects were present in the system. However, antisite defects are common when III-V semiconductor films are grown by MBE at the low temperatures required to prevent $\mathrm{Mn}$ segregation. The hole density and the Mn density are therefore taken as separate sample-dependent quantities, to be determined experimentally. The Hamiltonian of this model is 
specified in detail in the following paragraph. There has also been theoretical work on these materials based on a still simpler model [23] where holes are assumed to hop only between Mn acceptor sites, where they interact with the Mn moments via phenomenological exchange interactions. These models have some advantages in getting at the physics of the dilute Mn limit, and can also easily be adapted to include the holes that are localized on ionized antisite defects rather than Mn acceptors [24].

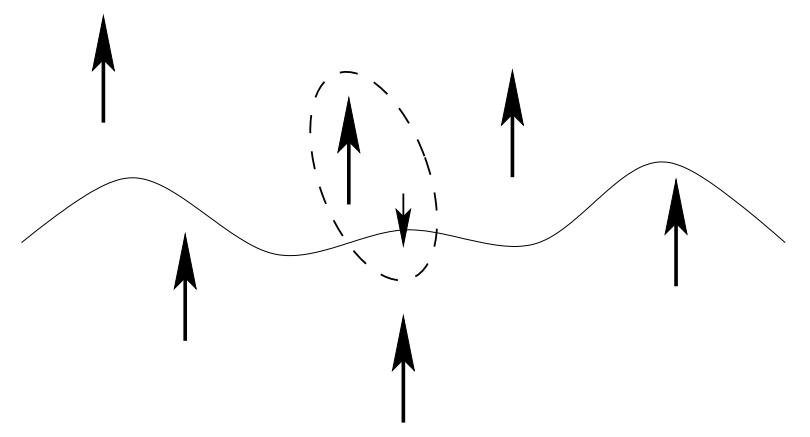

Fig. 1. Model for (III,Mn)V semiconductors: local magnetic moments $\left(\mathrm{Mn}^{2+}\right)$ with spin $S=5 / 2$ are antiferromagnetically coupled to itinerant carriers (holes) with spin $s=1 / 2$.

Like any phenomenological model, the one we use is defined most fundamentally by its low-energy degrees of freedom. Also important, however, is the Hamiltonian that acts in the implied Hilbert space. The length scales associated with holes in these compounds are still long enough that a $\boldsymbol{k} \cdot \boldsymbol{p}$, envelope function, description [25] of the semiconductor valence bands is appropriate and we take that approach here. The operators in terms of which the phenomenological Hamiltonian is expressed include the spin operator $\boldsymbol{S}_{I}$ for the $S=5 / 2$ local moment on site $I$ and the multi-band envelope function hole spin density operator $s(\boldsymbol{r})$. The following key terms are included in the minimal version of the model Hamiltonian:

a) The coupling of the Mn spin to the external magnetic field, $g \mu_{B} \sum_{I} \boldsymbol{S}_{I}$. $\boldsymbol{H}_{\text {ext }}$.

b) The band Hamiltonian of the host III-V semiconductor, usually described using a multi-band envelope function formalism [25,26]. For many properties it is necessary to incorporate spin-orbit coupling in a realistic way. Six- or eight-band models that include the 'split-off' band and/or the conduction band are sometimes desirable. Unlike the local moment models, the Hilbert space includes all host lattice sites for each hole rather than only localized orbitals centered on the Mn sites. This band Hamiltonian should include the strain effects due to lattice matching between 
the epitaxially grown (III,Mn)V films and the substrate on which they are grown.

c) Antiferromagnetic exchange coupling between the $\mathrm{Mn}^{2+}$ spin and valenceband holes, $J_{\mathrm{pd}} \sum_{I} \boldsymbol{S}_{I} \cdot \boldsymbol{s}\left(\boldsymbol{R}_{I}\right)$. This interaction represents virtual coupling to states that have been integrated out of the model's Hilbert space, ones in which electrons are exchanged between the Mn ion d shells and the valence band 21,22. The exchange interactions are isotropic to a good approximation because the $\mathrm{Mn}^{2+}$ ion has total angular momentum $L=0$. Experimental estimates for $J_{\mathrm{pd}}$ vary from $150 \pm 40 \mathrm{meV} \mathrm{nm}^{3}$ [1], to $68 \pm 10 \mathrm{meV} \mathrm{nm}^{3}$ [27, to $55 \pm 10 \mathrm{meV} \mathrm{nm}^{3}$ [11]. Recent experimental work limits $J_{p d}$ to a value toward the lower end of this range and fixes its value within perhaps $20 \%$.

The terms d)-f), listed below, are necessary to describe the crossover to the localized limit in which holes are bound either to Mn acceptors or to other defects. Note that simpler, impurity-band models assume that the system is in this limit from the outset, much as our model assumes from the outset that the Mn d-shells form local moments. There are sometimes technical difficulties in describing the localized limit with our higher-level model, so that considerable simplification arises from using the impurity-band model. There is however, a penalty to pay since the model does not apply to the regimes of greatest interest in which the band electrons are not localized. Even when impurity models do apply, it is difficult to guess at appropriate distribution functions for the inter-site hopping parameters that play a key role.

d) The attractive Coulomb interaction between the ionized $\mathrm{Mn}^{2+}$ acceptor and a valence-band hole. In an envelope function formalism, central-cell corrections to the interaction are necessary to capture the isolated boundacceptor limit accurately [28].

e) The repulsive Coulomb interaction among holes. This interaction is key in screening the ionized $\mathrm{Mn}^{2+}$ acceptors and cannot be neglected except in the completely-localized-hole limit. When it is included, it usually must be approximated in a way which avoids artificial hole-hole selfinteractions.

f) The repulsive interaction between holes and ionized antisite (group- $V$ element on group-III site) defects. The antisite defects compensate for the $\mathrm{Mn}$ acceptors and reduce the overall hole density, in addition to providing an important additional scattering center. In the dilute Mn limit, experiment [29] suggests that most $\mathrm{Mn}^{2+}$ ions do not have bound holes, possibly due to this compensation. When all holes are strongly localized, most Mn local moments will be free and the system will not have ferromagnetic order.

The following terms are potentially important in some circumstances. 
g) The scalar scattering potential that represents the energy difference between a valence-band $p$ electron on a host site and a valence-band $p$ electron on a Mn site. This effect has normally been excluded since its size and sign is not yet know.

h) Direct exchange interactions between $\mathrm{Mn}$ ions on neighboring sites. These terms result from microscopic processes in which exchange of electrons between the valence band and two nearby Mn d-shells is correlated. Terms of this type are known to be important in (II,Mn)VI semiconductors, but appear to be less important in (III,Mn)V semiconductors.

i) Direct coupling of band electrons to external magnetic field.

In the rest of this Chapter we discuss only pictures of (III,Mn) V ferromagnetism that follow from the minimal model that includes only the a)-c) Hamiltonian terms.

\section{Mean-field-theory predictions}

In this and the following section we make an important approximation that achieves a drastic simplification. We will refer to this as the continuum Mn approximation, although it has sometimes been referred to as a virtual crystal approximation. It is motivated by the observation that the Fermi wavelength of the valence-band electrons is typically longer than the distance between $\mathrm{Mn}$ ions, mainly because the Mn acceptors are compensated and also partially because there are four occupied valence bands. When the Mn ion distribution is replaced by a continuum with the same spin-density, randomness is completely eliminated from the minimal model. This approximation has many elements in common with the dynamic mean-field-theory (DMFT) approximation, applied 30 to these ferromagnets recently by Chattopadhyay et al., although the DMFT does retain some of the consequences of randomness neglected here and in the following section. The possibility of starting with a description based on an approximation where the random Mn distribution is replaced by a continuum emphasizes an essential difference between ferromagnetic semiconductors and classical spin glass systems in which dilute Mn local moments are distributed randomly in a metallic host. In both cases it is true that the interaction between local moments mediated by the itinerant electrons is oscillatory and ferromagnetic for separations smaller than the itinerant electron Fermi wavelength. However, the Fermi wavelength is longer than the distance between local moments in the doped semiconductor case, whereas it is shorter in the spin glass case [31]. Each Mn ion interacts ferromagnetically with several of its neighbors. The continuum Mn approximation will fail in the limit of dilute $\mathrm{Mn}$ ions and also if the exchange interaction between band and Mn spins is too strong. This approximation does not allow for the sensitivity of magnetic properties to annealing protocols that has been established in experiment. It does, however, seem to be reliable in the 
limit of principle interest, that of high Mn densities and high critical temperatures, where the holes are metallic and their interaction with Mn acceptors will be effectively screened. The merit of this approximation is that it enables quantitative prediction of many physical properties. In this section we discuss three important properties, the ferromagnetic transition temperature, the magnetic anisotropy energy, and the anomalous Hall conductance. This work described below is motivated by the view in science, it is ultimately up to experiment to decide on the reliability of any approximation made in modeling a physical system. As we will point out, the utility of the continuum Mn approximation is strongly supported by observations. In fact, it is not a surprise that this approximation is a good starting point, given its success in describing the influence of external fields on the properties of the closely related paramagnetic (II,Mn)VI semiconductors [20,22]. The present section makes in addition a mean-field approximation by ignoring correlations between Mn and band spin configurations.

Our mean-field theory is derived in the spin-density-functional framework and leads to a set of physically transparent coupled equations [32]. The effective magnetic field seen by localized magnetic ions consists of an external magnetic field and the mean kinetic-exchange-coupling contribution from spin-polarized carriers,

$$
\boldsymbol{H}_{\text {eff }}\left(\boldsymbol{R}_{I}\right)=\boldsymbol{H}_{\text {ext }}+J_{\mathrm{pd}}\left\langle\boldsymbol{s}\left(\boldsymbol{R}_{I}\right)\right\rangle / g \mu_{B},
$$

where $\left\langle\boldsymbol{s}\left(\boldsymbol{R}_{I}\right)\right\rangle$ is the carrier spin density at Mn sites, and $g$ is the g-factor of the local moments. The mean spin polarization of a magnetic ion is given by 33

$$
\langle\boldsymbol{S}\rangle_{I}=-S B_{S}\left(S g \mu_{B} H_{\mathrm{eff}}\left(\boldsymbol{R}_{I}\right) / k_{B} T\right) \hat{H}_{\mathrm{eff}}\left(\boldsymbol{R}_{I}\right),
$$

where $B_{S}(x)$ is the Brillouin function and $\hat{H}_{\text {eff }}\left(\boldsymbol{R}_{I}\right)$ is the unit vector along the direction of the effective magnetic field defined in Eq. (11). The itineranthole spin density is determined by solving the Schrödinger equation for holes which experience a kinetic-exchange effective Zeeman field $\boldsymbol{h}(\boldsymbol{r})$. The field $\boldsymbol{h}(\boldsymbol{r})$ is non-zero only in the ferromagnetic state and, in the continuum limit, reads

$$
\boldsymbol{h}(\boldsymbol{r})=J_{\mathrm{pd}} N_{\mathrm{Mn}}(\boldsymbol{r})\langle\boldsymbol{S}\rangle(\boldsymbol{r}),
$$

where $N_{M n}=4 x / a_{l c}^{3}$ is the Mn density in $\mathrm{Mn}_{x} \mathrm{III}_{1-x} \mathrm{~V}$ zincblende semiconductors with a lattice constant $a_{l c}$. For inhomogeneous systems such as quantum wells or superlattices, the itinerant holes experience also an electrostatic potential due to heterostructure confinement and external bias (if present). Using the local-spin-density approximation (LSDA), itinerant hole-hole interaction can be accounted for by including an additional spin-dependent one-particle potential in the Schrödinger equation. 


\subsection{Ferromagnetic transition temperature}

In homogeneous DMS systems, the hole-spin density $\langle s\rangle$ and the kineticexchange potential $h$ are related at small $h$ by

$$
\langle s\rangle=-\frac{\chi_{f}}{\left(g^{*} \mu_{B}\right)^{2}} h .
$$

Here, $g^{*}$ is the hole g-factor and $\chi_{f}$ is the interacting hole magnetic susceptibility,

$$
\frac{\chi_{f}}{\left(g^{*} \mu_{B}\right)^{2}}=-\frac{d^{2}\left(E_{\mathrm{tot}} / V\right)}{d h^{2}},
$$

where $E_{\text {tot }} / V$ is the total energy density of the itinerant-hole system. The Curie-Weiss transition temperature, obtained from Eqs. (11) - (何) in the $H_{\text {ext }}=0$ limit, is

$$
k_{B} T_{c}=\frac{N_{\mathrm{Mn}} S(S+1)}{3} \frac{J_{\mathrm{pd}}^{2} \chi_{f}}{\left(g^{*} \mu_{B}\right)^{2}} .
$$

To understand the qualitative physics implicit in this $T_{c}$-equation (6), we discuss first the magnetic susceptibility expressions of a model itinerant electron system with a single spin-split band and an effective mass $m^{*}$. The kinetic-energy contribution $E_{\text {tot }}^{\text {kin }}$ to the total energy gives

$$
\frac{d^{2}\left(E_{\mathrm{tot}}^{\mathrm{kin}} / V\right)}{d h^{2}}=-\frac{m^{*} k_{F}}{4 \pi^{2} \hbar^{2}},
$$

where $k_{F}$ is the Fermi wavevector. The exchange energy of the spin-polarized parabolic-band model adds a contribution

$$
\frac{d^{2}\left(E_{\mathrm{tot}}^{\mathrm{exch}} / V\right)}{d h^{2}}=-\frac{e^{2}\left(m^{*}\right)^{2}}{4 \pi^{3} \varepsilon \hbar^{4}},
$$

where $\varepsilon$ is the dielectric constant of the host semiconductor. At high hole densities $p$, the kinetic-energy term dominates and $T_{c}$ is proportional to the Fermi wavevector, i.e., to $p^{1 / 3}$. Equations (7) and (8) also show that the band contribution to the mean-field $T_{c}$ increases linearly with $m^{*}$ while the exchange enhancement of $T_{c}$ is proportional to $\left(m^{*}\right)^{2}$. Note that correlation effects, not discussed here in detail, suppress the mean-field $T_{c}$ by only $\sim 1 \%$ for typical experimental hole densities $\left(p \sim 0.1 \mathrm{~nm}^{-3}\right)$ in bulk (III,Mn)As ferromagnets.

To obtain quantitative predictions for $T_{c}$, it is necessary to evaluate the kinetic and exchange contribution to the itinerant hole susceptibility using a realistic six-band Kohn-Luttinger model [26], instead of the parabolic band model. The Hamiltonian contains the spin-orbit splitting parameter $\Delta_{\text {so }}$ and three other phenomenological parameters, $\gamma_{1}, \gamma_{2}$, and $\gamma_{3}$, whose values for the specific III-V host can be found, e.g., in Refs. [7, 34]. Results [7, 35, 36, 37] 


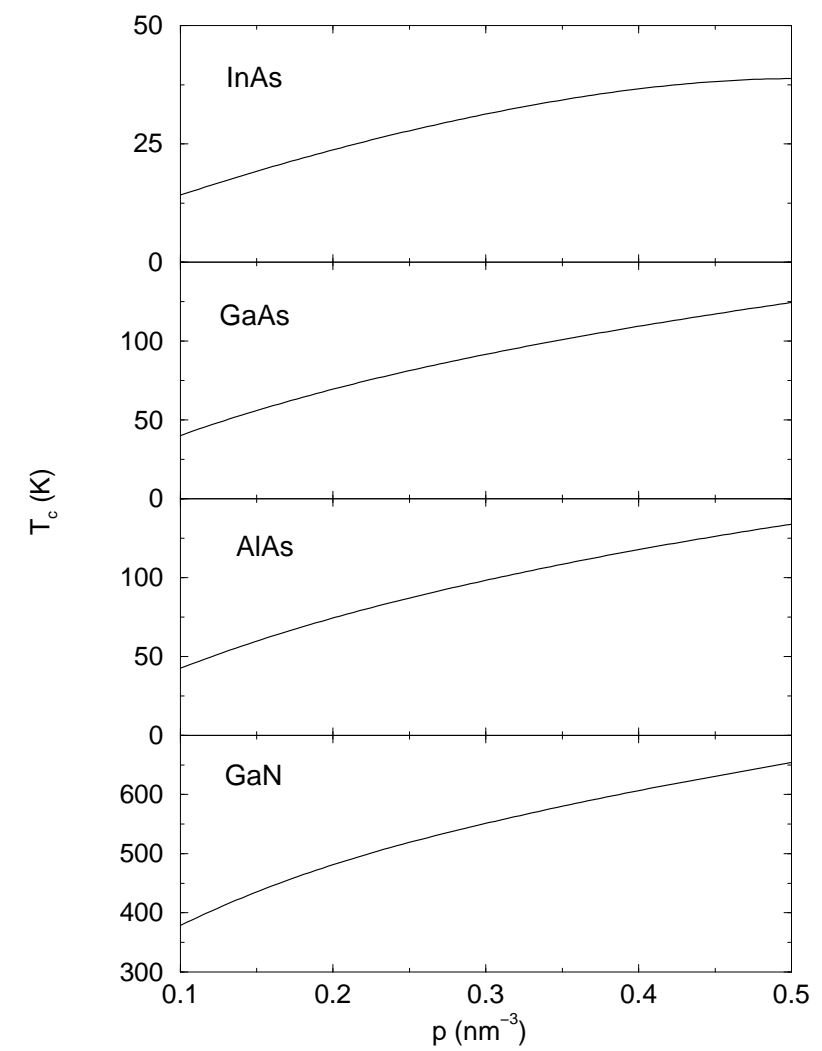

Fig. 2. The band (kinetic energy) contribution to the mean-field ferromagnetic critical temperature $T_{c}$ for Mn concentration $x=5 \%$ is plotted as a function of hole density $p$ for InAs, GaAs, AlAs, and GaN host semiconductors.

are plotted in Fig. 2 as a function of the hole density for InAs, GaAs, AlAs, and $\mathrm{GaN}$ host semiconductors. In the density range considered, only the two heavy-hole and two light-hole bands are occupied in the arsenides. However, the mixing between these four bands and the two spin-orbit split-off bands is strong and must be accounted for. In GaN, spin-orbit coupling is weak and all six bands are occupied by holes. The numerical data are consistent with the qualitative analysis based on the parabolic band model: the band (kinetic-energy) contribution to $T_{c}$ follows roughly the $p^{1 / 3}$ dependence, the exchange enhancement of $\sim 10 \%$ is only weakly density dependent. The $T_{c}$ values at a given density are ordered according to the heavy-hole and lighthole masses in the arsenide hosts. For GaN, with all six bands occupied, the simple model of a parabolic spin-split band is less instructive. Yet the large numerical $T_{c}$ 's in this material are consistent with the large heavy-hole mass, nearly twice as large as in AlAs. 
The mean-field prediction for the critical temperature agrees quantitatively with the experimental value of $110 \mathrm{~K}$ measured in Mn-doped GaAs with Mn concentration $x=5 \%$ and $p=0.35 \mathrm{~nm}^{-3}$. Thermal fluctuations neglected by the mean-field theory, discussed in the following section, reduce the theoretical $T_{c}$ estimate by less than $5 \%$ [37], explaining the quantitative success of the mean-field theory in this sample. The same analysis finds approximately a $T_{c}$ suppression [37] of approximately $20 \%$ compared to mean-field theory due for $(\mathrm{Ga}, \mathrm{Mn}) \mathrm{N}$, implying that room temperature ferromagnetism may occur in III-V DMS.

\subsection{Magnetic anisotropy}

Experiments [14,38 in (III,Mn)V DMS's have demonstrated that these ferromagnets have remarkably square hysteresis loops and that the magnetic easy axis is dependent on epitaxial growth lattice-matching strains. The physical origin [7.26] of the anisotropy energy in our model is spin-orbit coupling in the valence band. Even in mean-field theory, we find that the magnetic anisotropy physics of these materials is rich and that easy axis reorientations can occur as a function of sample parameters including hole density or epitaxial growth lattice-matching strains.

Magnetic anisotropy in the absence of strain is well described by a cubic harmonic expansion truncated at sixth order, an approximation commonly used in the literature [39] on magnetic materials. The corresponding cubic harmonic expansion for total energy of a system of non-interacting holes in the presence of the effective field $h$ is

$$
\frac{E_{\mathrm{tot}}(\hat{M})}{V}=\frac{E_{\mathrm{tot}}(\langle 100\rangle)}{V}+K_{1}^{\mathrm{ca}}\left(\hat{h}_{x}^{2} \hat{h}_{y}^{2}+\hat{h}_{y}^{2} \hat{h}_{z}^{2}+\hat{h}_{x}^{2} \hat{h}_{z}^{2}\right)+K_{2}^{\mathrm{ca}} \hat{h}_{x}^{2} \hat{h}_{y}^{2} \hat{h}_{z}^{2},
$$

where $\hat{h}$ is the unit vector along the field $h$. The cubic anisotropy coefficients $K_{1}^{\text {ca }}$ and $K_{2}^{\text {ca }}$ are related to total energies for $\hat{h}$ along the high symmetry crystal directions by following expressions:

$$
\begin{aligned}
K_{1}^{\mathrm{ca}} & =\frac{4\left[E_{\mathrm{tot}}(\langle 110\rangle)-E_{\mathrm{tot}}(\langle 100\rangle)\right]}{V} \\
K_{2}^{\mathrm{ca}} & =\frac{27 E_{\mathrm{tot}}(\langle 111\rangle)-36 E_{\mathrm{tot}}(\langle 110\rangle)+9 E_{\mathrm{tot}}(\langle 100\rangle)}{V} .
\end{aligned}
$$

MBE growth techniques produce (III,Mn)V films whose lattices are locked to those of their substrates. X-ray diffraction studies [5] have established that the resulting strains are not relaxed by dislocations or other defects, even for thick films. Strains in the (III,Mn)V film break the cubic symmetry assumed in Eq. (9). However, the influence of MBE growth lattice-matching strains on the hole bands of cubic semiconductors is well understood 25] and we can use the same formal mean-field theory as in the previous subsection to account for strain effects on magnetic anisotropy. 


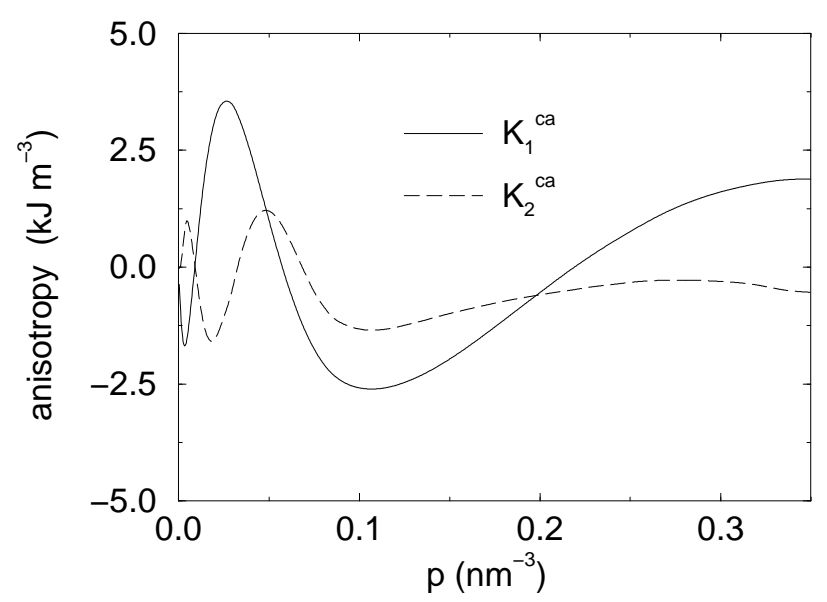

Fig. 3. Cubic magnetic anisotropy coefficients $K_{1}^{\mathrm{ca}}$ and $K_{2}^{\mathrm{ca}}$ as a function of hole density $p$.

We turn now to a series of illustrative calculations intended to closely model the ground state of (Ga,Mn)As. For Mn density $N_{\mathrm{Mn}}=1 \mathrm{~nm}^{-3}$ $(x \approx 5 \%), h \approx 140 \mathrm{meV}$ at zero temperature. This value of $h$ is not so much smaller than the spin-orbit splitting parameter in GaAs [34.] $\left(\Delta_{\mathrm{so}}=\right.$ $341 \mathrm{meV}$ ), so that accurate calculations require the six-band Luttinger model [26. Even with $N_{\mathrm{Mn}}$ fixed, our calculations show that the magnetic anisotropy of (III,Mn)V ferromagnets is strongly dependent on both hole density and strain. The hole density can be varied by changing growth conditions or by adding other dopants to the material, and strain in a (Ga,Mn)As film can be altered by changing substrates. The cubic anisotropy coefficients (in units of energy per volume) for strain-free material are plotted as a function of hole density in Fig. 3. The easy axis is nearly always determined by the leading cubic anisotropy coefficient $K_{1}^{\text {ca }}$, except near values of $p$ where this coefficient vanishes. As a consequence, the easy axis in strain free samples is almost always either along one of the cube edge directions $\left(K_{1}^{\mathrm{ca}}>0\right)$, or along one of the cube diagonal directions $\left(K_{1}^{\text {ca }}<0\right)$. Transitions in which the easy axis moves between these two directions occur twice over the range of hole densities studied. (Similar transitions occur as a function of $h$, and therefore temperature, for fixed hole density.) Near the hole density $p=0.01 \mathrm{~nm}^{-3}$, both anisotropy coefficients nearly vanish and a fine-tuned nearly perfect isotropy is achieved. The slopes of the anisotropy coefficient curves vary as the number of occupied bands increases from 1 to 4 with increasing hole density. This behavior is clearly seen from the correlation between oscillations of the anisotropy coefficients and onsets of higher band occupations.

Six-band model Fermi surfaces are illustrated in Figs. 1 and 5 by plotting their intersections with the $k_{z}=0$ plane at $p=0.1 \mathrm{~nm}^{-3}$ for the cases of 
$\langle 100\rangle$ and $\langle 110\rangle$ ordered moment orientations. The dependence of quasiparticle band structure on ordered moment orientation, apparent in comparing these figures, should lead to large anisotropic magnetoresistance effects in (Ga,Mn)As ferromagnets. We also note that in the case of cube edge orientations, the Fermi surfaces of different bands intersect. This property could have important implications for the decay of long-wavelength collective modes.

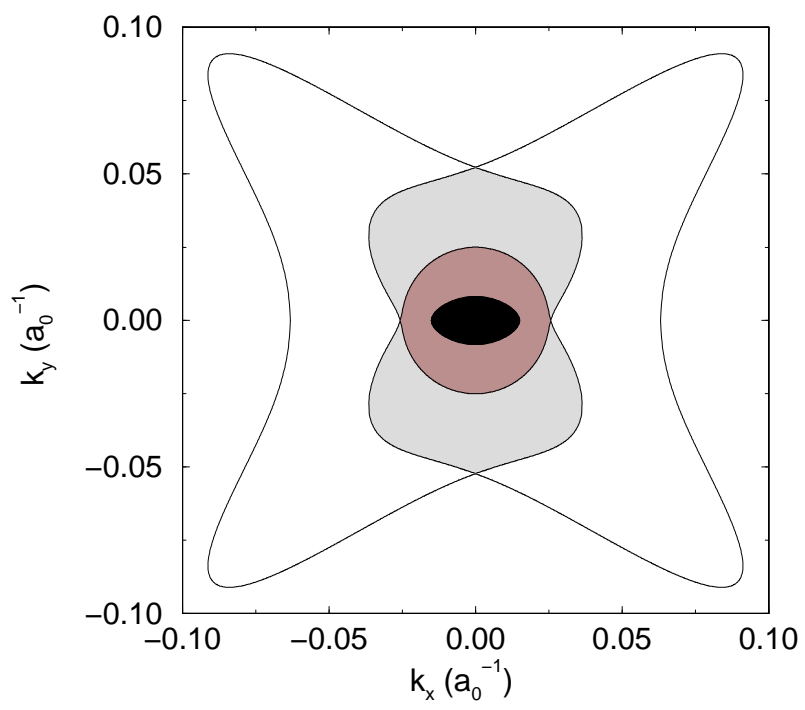

Fig. 4. Six-band model Fermi surface intersections with the $k_{z}=0$ plane for $p=$ $0.1 \mathrm{~nm}^{-3}$ and $h=140 \mathrm{meV}$. This figure is for magnetization orientation along the $\langle 100\rangle$ direction.

In Fig. 6 we present mean-field theory predictions for the strain-dependence of the anisotropy energy at $h=140 \mathrm{meV}$ and hole density $p=0.35 \mathrm{~nm}^{-3}$. According to our calculations, the easy axes in the absence of strain are along the cube edges in this case. The relevant value of the in-plane strain produced by the substrate-film lattice mismatch,

$$
e_{0}=\frac{a_{s}-a_{f}}{a_{f}}
$$

depends on the substrate on which the epitaxial (Ga,Mn)As film is grown. The most important conclusion from Fig. 6 is that strains as small as $1 \%$ are sufficient to completely alter the magnetic anisotropy energy landscape. For example for $(\mathrm{Ga}, \mathrm{Mn}) \mathrm{As}$ on GaAs, $e_{0}=-0.0028$ at $x=0.05$. The anisotropy has a relatively strong uniaxial contribution, even for this rel- 


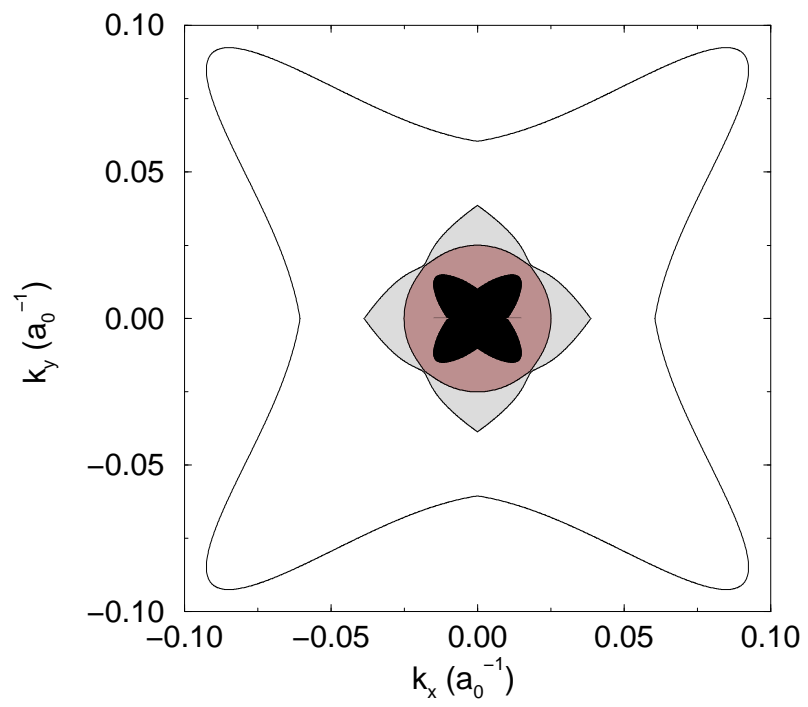

Fig. 5. Six-band model Fermi surface intersections with the $k_{z}=0$ plane for the parameters of Fig. $\square$ and magnetization orientation along the $\langle 110\rangle$ direction.

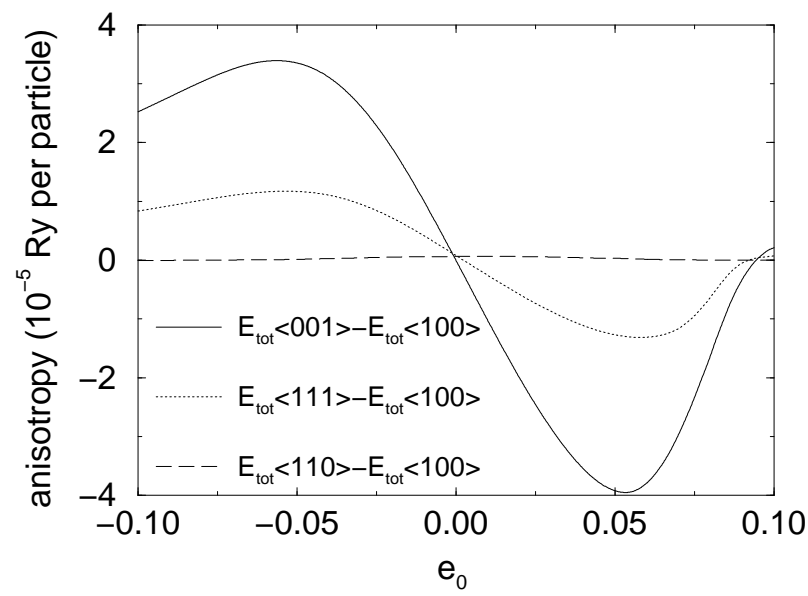

Fig. 6. Energy differences among $\langle 001\rangle,\langle 100\rangle,\langle 110\rangle$, and $\langle 111\rangle$ magnetization orientations vs. in-plane strain $e_{0}$ at $h=140 \mathrm{meV}$ and $p=0.35 \mathrm{~nm}^{-3}$. For compressive strains $\left(e_{0}<0\right)$, the system has an easy magnetic plane perpendicular to the growth direction. For tensile strains $\left(e_{0}>0\right)$, the anisotropy is easy-axis with the preferred magnetization orientation along the growth direction. The anisotropy changes sign at large tensile strain. 
atively modest compressive strain, which favors in-plane moment orientations [7,26], in agreement with experiment [5]. A relatively small $(\sim 1 \mathrm{~kJ}$ $\mathrm{m}^{-3}$ ) residual in-plane anisotropy remains which favors $\langle 110\rangle$ over $\langle 100\rangle$. For $x=0.05(\mathrm{Ga}, \mathrm{Mn})$ As on a $x=0.15$ (In,Ga)As buffer the strain is tensile, $e_{0}=0.0077$, and we predict a substantial uniaxial contribution to the anisotropy energy which favors growth direction orientations [7.,26], again in agreement with experiment [5]. For the tensile case, the anisotropy energy changes more dramatically than for compressive strains due to the depopulation of higher subbands. At large tensile strains, the sign of the anisotropy changes, emphasizing the subtlety of these effects and the latitude which exists for strain-engineering of magnetic properties.

\subsection{Anomalous Hall effect}

The mean-field description of hole bands in the presence of exchange coupling to the localized $\mathrm{Mn}$ moments provides a starting point for building a theory of transport in (III,Mn)V ferromagnets. Here we concentrate on the anomalous Hall effect which is an important sample characterization tool in ferromagnetic systems. The Hall resistivity of ferromagnets has an ordinary contribution, proportional to the external magnetic-field strength, and an anomalous contribution usually assumed to be proportional to the sample magnetization. In our approach 40, the anomalous Hall conductance of a homogeneous ferromagnet is related to the Berry phase of the electronic wavefunction acquired by a cyclic evolution along the Fermi surface.

In the standard model of the AHE in metals, skew-scattering 44] and side-jump [42] scattering give rise to contributions to the Hall resistivity proportional to the diagonal resistivity $\rho$ and $\rho^{2}$ respectively, with the latter process tending to dominate in alloys because $\rho$ is larger. Our evaluation of the AHE in (III,Mn)V ferromagnets is based on a theory 43 of semiclassical wave-packet dynamics which implies a contribution to the Hall conductivity that is independent of the kinetic-equation scattering term. The interest in this contribution is motivated in part by practical considerations, since our current understanding of (III,Mn) V ferromagnets is not sufficient to permit confident modeling of quasiparticle scattering. The relation of our approach to standard theory is reminiscent of disagreements between Smit 41 and Luttinger 44 that occurred early in the development of AHE theory and do not appear to have ever been fully resolved. We follow Luttinger [44] in taking the view that there is a contribution to the AHE due to the change in wavepacket group velocity that occurs when an electric field is applied to a ferromagnet. The electron group velocity correction is conveniently evaluated using expressions derived by Sundaram and Niu [43]:

$$
\dot{\boldsymbol{x}}_{c}=\frac{\partial \epsilon}{\hbar \partial \boldsymbol{k}}+(e / \hbar) \boldsymbol{E} \times \boldsymbol{\Omega} .
$$

The first term on the right-hand-side of Eq. (12) is the standard Bloch band group velocity. Our anomalous Hall conductivity is due to the second term, 
proportional to the Berry curvature $\boldsymbol{\Omega}$, defined below. It follows from symmetry considerations that for a cubic semiconductor under lattice-matching strains and with $\hat{m}$ aligned by external fields along the $\langle 001\rangle$ growth direction, only the $z$-component of $\boldsymbol{\Omega}$ is nonzero:

$$
\Omega_{z}(n, \boldsymbol{k})=2 \operatorname{Im}\left[\left\langle\frac{\partial u_{n}}{\partial k_{y}} \mid \frac{\partial u_{n}}{\partial k_{x}}\right\rangle\right] .
$$

Here $\left|u_{n}\right\rangle$ is the periodic part of the $n$-th Bloch band wavefunction with the mean-field spin-splitting term included in the Hamiltonian. The anomalous Hall conductivity that results from this velocity correction is

$$
\sigma_{\mathrm{AH}}=-\frac{e^{2}}{\hbar} \sum_{n} \int \frac{d \boldsymbol{k}}{(2 \pi)^{3}} f_{n, \boldsymbol{k}} \Omega_{z}(n, \boldsymbol{k}),
$$

where $f_{n, \boldsymbol{k}}$ is the equilibrium Fermi occupation factor for the band quasiparticles. We have taken the convention that a positive $\sigma_{\mathrm{AH}}$ means that the anomalous Hall current is in the same direction as the normal Hall current.

This Berry phase contribution to the anomalous Hall conductance occurs in any itinerant electron ferromagnet with spin-orbit coupling. To assess its importance for (III,Mn)V ferromagnets we first explore a simplified model that yields parabolic dispersions for the two heavy-hole and two light-hole bands, and neglect coupling to the split-off band by assuming a large spinorbit coupling 25,26. Detailed numerical simulations accounting for the mixing of the spin-orbit split-off bands and warping of the occupied heavy-hole and light-hole bands [25,26] in the (In,Mn)As and (Ga,Mn)As samples [45, 1] will follow. Within the 4-band spherical model, the spin operator $s=\boldsymbol{j} / 3$, and the Hamiltonian for holes in III-V host semiconductors can be written as

$$
H_{0}=\frac{\hbar^{2}}{2 m}\left[\left(\gamma_{1}+\frac{5}{2} \gamma_{2}\right) k^{2}-2 \gamma_{2}(\boldsymbol{k} \cdot \boldsymbol{j})^{2}\right],
$$

where $\boldsymbol{j}$ is the total angular momentum operator and $\gamma_{1}$ and $\gamma_{2}$ are the Luttinger parameters 25,34. In the unpolarized case $(h=0)$, the total Hamiltonian, $H=H_{0}-h j_{z} / 3$ (the external magnetic field is assumed to be in the $+\hat{z}$ direction), is diagonalized by spinors $\left|j_{\hat{k}}\right\rangle$ where, e.g., $j_{\hat{k}} \equiv \boldsymbol{j} \cdot \hat{k}= \pm 3 / 2$ for the two degenerate heavy-hole bands with the effective mass $m_{\mathrm{hh}}=m /\left(\gamma_{1}-2 \gamma_{2}\right)$. The corresponding Berry phase, $\int d^{2} k \Omega( \pm 3 / 2, \boldsymbol{k})= \pm 3 / 2\left(\cos \theta_{\boldsymbol{k}}-1\right)$, is largest at the equator $\left(\cos \theta_{\boldsymbol{k}} \equiv k_{z} / k_{\mathrm{hh}}=0\right)$ and vanishes at the poles $\left(\left|\cos \theta_{\boldsymbol{k}}\right|=1\right)$ of the spherical Fermi surface of radius $k_{\mathrm{hh}}$. Because of the band degeneracy, the anomalous Hall conductivity (14) vanishes in the $h=0$ limit. The effective Zeeman coupling present in the ferromagnetic state both modifies the Fermi surface shapes and renormalizes the Berry phases. Up to linear order in $h$ we obtain that $k_{\mathrm{hh}}^{ \pm}=k_{\mathrm{hh}} \pm h m_{\mathrm{hh}} /\left(2 \hbar^{2} k_{\mathrm{hh}}\right) \cos \theta_{\boldsymbol{k}}$ and that the Berry phase is reduced (enhanced) by a factor $\left[1 \mp 2 m h /\left(9 \gamma_{2} \hbar^{2} k_{\mathrm{hh}}^{2}\right)\right]$. A 
similar analysis for the light-hole bands leads to the total net contribution to the AHE from the four bands whose lower and upper bounds are:

$$
\frac{e^{2}}{4 \pi^{2} \hbar^{3}} h\left(3 \pi^{2} p\right)^{-1 / 3} m_{\mathrm{hh}}<\sigma_{\mathrm{AH}}<\frac{e^{2}}{4 \pi^{2} \hbar^{3}} h\left(3 \pi^{2} p\right)^{-1 / 3} 2^{2 / 3} m_{\mathrm{hh}} .
$$

Here $p=k_{\mathrm{hh}}^{3} / 3 \pi^{2}\left(1+\sqrt{m_{\mathrm{lh}} / m_{\mathrm{hh}}}\right)$ is the total hole density and $m_{\mathrm{lh}}=$ $m /\left(\gamma_{1}+2 \gamma_{2}\right)$ is the light-hole effective mass. The lower bound in Eq. (16) is obtained assuming $m_{\mathrm{lh}} \ll m_{\mathrm{hh}}$ while the upper bound is reached when $m_{\mathrm{lh}} \approx m_{\mathrm{hh}}$.

Based on the above analysis we draw the following conclusions: The anomalous velocity due to the Berry phase can have a sizable effect on the AHE in (III,Mn)V ferromagnets. The solid line in Fig. 1 shows our analytic results for the GaAs effective masses $m_{\mathrm{hh}}=0.5 m_{e}$ and $m_{\mathrm{lh}}=0.08 m_{e}$. Note that in experiment, anomalous Hall conductances are in order of 1-10 $\Omega^{-1} \mathrm{~cm}^{-1}$ and the effective exchange field $h \sim 10-100 \mathrm{meV}$. A large $\sigma_{\mathrm{AH}}$ is expected in systems with large heavy-hole effective mass and with the ratio $m_{\mathrm{lh}} / m_{\mathrm{hh}}$ close to unity.

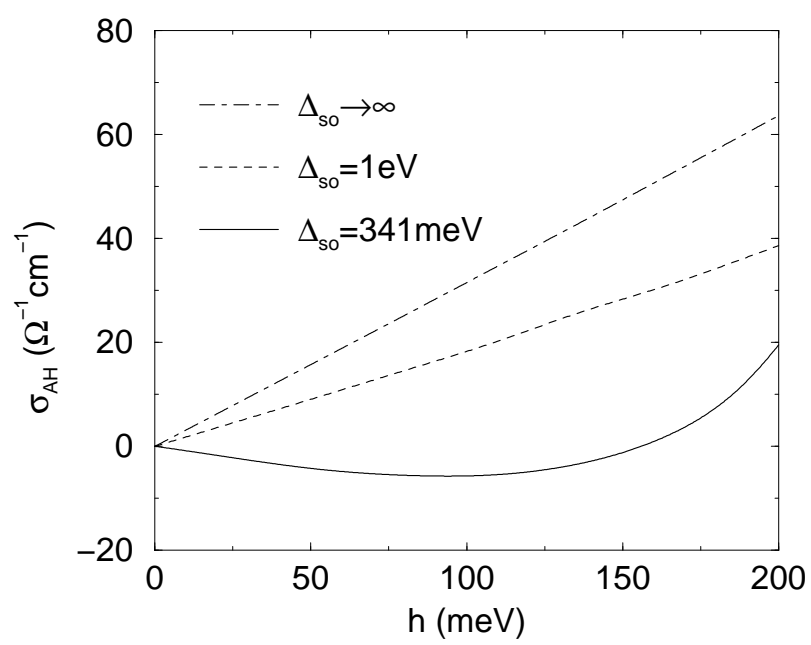

Fig. 7. Illustrative calculations of the anomalous Hall conductance as a function of the band-splitting effective Zeeman field for hole density $p=0.35 \mathrm{~nm}^{-1}$. The dotted-dashed curve was obtained assuming infinitely large spin-orbit coupling and the decrease of theoretical $\sigma_{\mathrm{AH}}$ with decreasing spin-orbit coupling strength is demonstrated for $\Delta_{\text {so }}=1 \mathrm{eV}$ (dashed line) and $\Delta_{\text {so }}=341 \mathrm{meV}$ (solid line).

So far we have discussed the limits of infinitely strong spin-orbit coupling and weak effective exchange field, relative to the hole Fermi energy. In the opposite limits of zero spin-orbit coupling or large $h, \sigma_{\mathrm{AH}}$ vanishes. This implies that the anomalous Hall conductivity is generally nonlinear in the 
exchange field or the magnetization. To explore the intermediate regime we diagonalized the six-band Luttinger Hamiltonian numerically [25:26] with the spin-orbit gap $\Delta_{\text {so }}=1 \mathrm{eV}$ as well as for the GaAs value $\Delta_{\text {so }}=341 \mathrm{meV}$. Results shown in Fig. 7 confirm that a smaller $\sigma_{\mathrm{AH}}$ is expected in systems with smaller $\Delta_{\text {so }}$ and suggest that both positive and negative signs of $\sigma_{\mathrm{AH}}$ can occur, in general. The curves in Fig. 7 are obtained by neglecting band warping in III-V semiconductor compounds. The fact that valence bands in these materials are typically strongly non-parabolic, even in the absence of the field $h$ and in the large $\Delta_{\text {so }}$ limit, is accurately captured by introducing the third phenomenological Luttinger parameter $\gamma_{3}$ [25,26]. Numerical data including all Luttinger parameters indicates that warping tends to lead to an increase of $\sigma_{\mathrm{AH}}$, as seen when comparing solid curves in Fig. 7 and in the top panel of Fig. 8. The hole-density dependence of $\sigma_{\mathrm{AH}}$, illustrated in Fig. 8, is qualitatively consistent with the spherical model prediction (16). Also in accord with the outlined chemical trends, numerical data in Fig. 8 suggest large positive AHE in (Al,Mn)As, intermediate positive $\sigma_{\mathrm{AH}}$ in $(\mathrm{Ga}, \mathrm{Mn}) \mathrm{As}$, and a relatively weaker AHE in (In,Mn)As with the sign of $\sigma_{\mathrm{AH}}$ that may depend on the detail structure of the sample.

We make now a comparison between our $\sigma_{\mathrm{AH}}$ calculations and experimental data in the (In,Mn)As and (Ga,Mn)As samples, analyzed in detail by Ohno and coworkers 45, 1,5. The nominal Mn densities in the two measured systems are $N_{\mathrm{Mn}}=0.23 \mathrm{~nm}^{-3}$ for the InAs host and $N_{\mathrm{Mn}}=1.1 \mathrm{~nm}^{-3}$ for the GaAs host, yielding saturation values of the effective field $h=25 \pm 3 \mathrm{meV}$ and $h=122 \pm 14 \mathrm{meV}$, respectively. The low-temperature hole density of the (Ga,Mn)As sample, $p=0.35 \mathrm{~nm}^{-3}$, was unambiguously determined [5] from the ordinary Hall coefficient measured at high magnetic fields of 22-27 T. Since a similar experiment has not been reported for the (In,Mn)As sample we estimated the hole density, $p=0.1 \mathrm{~nm}^{-3}$, by matching the measured ferromagnetic transition temperature $T_{c}=7.5 \mathrm{~K}$ to the density dependent mean-field $T_{c}$.

As demonstrated in Fig. 8, our theory explains the order of magnitude difference between experimental AHE in the two samples $\left(\sigma_{\mathrm{AH}} \approx 1 \Omega^{-1} \mathrm{~cm}^{-1}\right.$ in $(\mathrm{In}, \mathrm{Mn}) \mathrm{As}$ and $\sigma_{\mathrm{AH}} \approx 14 \Omega^{-1} \mathrm{~cm}^{-1}$ in $\left.(\mathrm{Ga}, \mathrm{Mn}) \mathrm{As}\right)$. The calculations are also consistent with the observed positive sign and monotonic dependence of $\sigma_{\mathrm{AH}}$ on sample magnetizations [0].

We take the agreement in both magnitude and sign of the AHE as a strong indication that the anomalous velocity contribution dominates the AHE in homogeneous (III,Mn) V ferromagnets. This Berry phase term, which is independent of quasiparticle scatterers, is relatively easily evaluated with high accuracy, enhancing the utility of the Hall measurement in sample characterization. The success of this model also supports the use of the simple mean-field approximation discussed in this section, in which Mn ions are represented by a uniform density continuum, to describe at least the ground state of these ferromagnets. 


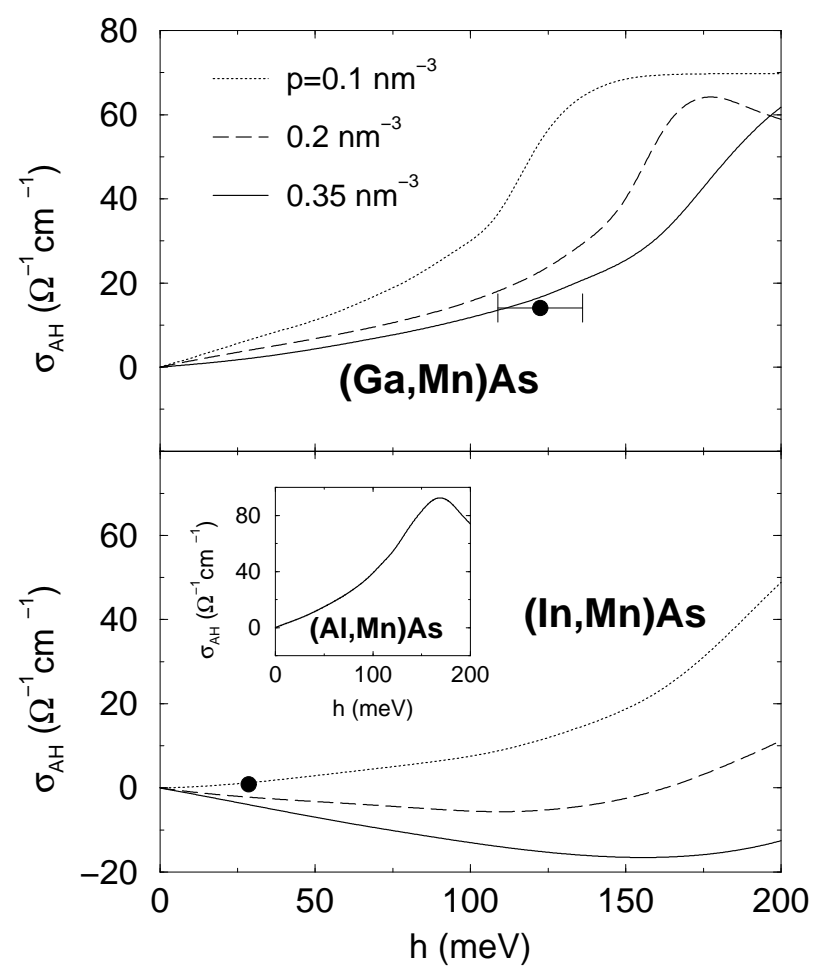

Fig. 8. Full numerical simulations of $\sigma_{\mathrm{AH}}$ for GaAs host (top panel), InAs host (bottom panel), and AlAs host (inset) with hole densities $p=0.1 \mathrm{~nm}^{-1}$ (dotted lines), $p=0.2 \mathrm{~nm}^{-1}$ (dashed lines), and $p=0.35 \mathrm{~nm}^{-1}$ (solid lines). The filled circles in the top and bottom panels represent measured AHE 45,5 values. The saturation mean-field $h$ values for the two points were estimated from nominal sample parameters 45, H. H. Horizontal error bars correspond to the experimental uncertainty of the $J_{\mathrm{pd}}$ coupling constant. The measured hole density in the (Ga,Mn)As sample is $p=0.35 \mathrm{~nm}^{-1}$; for (In,Mn)As, $p=0.1 \mathrm{~nm}^{-1}$ was determined indirectly from the sample's transition temperature.

\section{Collective excitations within a continuum picture}

\subsection{Beyond mean-field theory and RKKY interaction}

The power and the success of the mean-field picture employed in the previous section lies in the fact that it is a simple theoretical approach which makes it easy to calculate many observable quantities numerically. Mean-field theory, however, neglects correlation between local-moment spin configurations and the free-carrier state and, therefore, fails to describe the existence of low-energy long-wavelength spin excitations, among other things. Because of its neglect of collective magnetization fluctuations, mean-field theory, e.g., always overestimates the ferromagnetic critical temperature. There are many 
examples in itinerant electron systems where mean-field theory overestimates ferromagnetic transition temperatures by more than an order of magnitude and it is not a priori obvious that mean-field theory will be successful in (III,Mn)V ferromagnets. Indeed, we will find that the multi-band character of the semiconductor valence band plays an essential role in enabling high ferromagnetic transition temperatures in these materials.

In this section we identify the elementary spin excitations, determine their dispersion, and discuss implications for the Curie temperature $46,47,48,49,50$. The starting point of our analysis is the itinerant-carrier-mediated ferromagnetic interaction between local magnetic moments. Such an interaction is provided by the familiar Ruderman-Kittel-Kasuya-Yoshida (RKKY) theory. The RKKY picture, however, only applies as long as the perturbation induced by the Mn spins on the itinerant carriers is small. As we will derive below, the proper condition is $\Delta \ll \epsilon_{F}$ where $\Delta=N_{\mathrm{Mn}} J_{\mathrm{pd}} S$ is the (zero-temperature) spin-splitting gap of the itinerant carriers due to an average effective field induced by the Mn ions, and $\epsilon_{F}$ is the Fermi energy. This condition is, however, never satisfied in $(\mathrm{III}, \mathrm{Mn}) \mathrm{V}$ ferromagnets, partially because the valence-band carrier concentration $p$ is usually much smaller than the Mn impurity density $N_{\mathrm{Mn}}$. A related drawback of the RKKY picture is that it assumes an instantaneous static interaction between the magnetic ions, i.e., the dynamics of the free carriers are neglected. We will see below that this dynamics is important to obtain all types of elementary spin excitations. As a consequence, $R K K Y$ theory does not provide a proper description of the ordered state in ferromagnetic DMSs.

As in the previous section, we use here the minimal model including terms a) -c) of Section 3 and employ the Mn continuum approximation. Extensions to the minimal model may be important in some circumstances. They are, however, not essential for the general discussion in the present section, which will attempt to explain the considerations that determine when collective effects neglected by mean-field-theory are important.

\subsection{Independent spin-wave theory for parabolic bands}

The main idea of our theory is to derive an effective description for the Mn spin system by integrating out the valence-band carriers and to look for fluctuations of the Mn spins around their spontaneous mean-field magnetization direction (which we choose as the $z$-axis). Using the Holstein-Primakoff (HP) representation [51], we express the Mn spins in terms of bosonic degrees of freedom. We expand the effective action up to quadratic order, i.e., we treat the spin excitations as noninteracting Bose particles. From the corresponding propagator we deduce the dispersion of all elementary spin excitations.

To keep the discussion transparent we start with a two-band model for the itinerant carriers with quadratic dispersion. Later, in Section 5.5, we extend our theory to a model with a more realistic band structure described by a six-band Kohn-Luttinger Hamiltonian. 
For small fluctuations around the mean-field magnetization, we can write the spin operators as

$$
\begin{aligned}
S^{+}(\boldsymbol{r}) & \approx b(\boldsymbol{r}) \sqrt{2 N_{\mathrm{Mn}} S} \\
S^{-}(\boldsymbol{r}) & \approx b^{\dagger}(\boldsymbol{r}) \sqrt{2 N_{\mathrm{Mn}} S} \\
S^{z}(\boldsymbol{r}) & =N_{\mathrm{Mn}} S-b^{\dagger}(\boldsymbol{r}) b(\boldsymbol{r})
\end{aligned}
$$

with bosonic fields $b^{\dagger}(\boldsymbol{r}), b(\boldsymbol{r})$. The state with fully polarized Mn spins (along the $z$-direction) corresponds, in the HP boson language, to the vacuum with no bosons. The creation of a HP boson reduces the magnetic quantum number by one.

The partition function $Z$ can be expressed as a coherent-state path integral in imaginary time over the HP bosons and the valence-band carriers, which are fermions. Since the Hamiltonian is bilinear in the fermionic fields, we can integrate out the itinerant carriers and arrive at an effective description in terms of the impurity spin degree of freedom labeled by the complex number coherent state labels for the boson fields, $z$ and $\bar{z}$. We get $Z=\int \mathcal{D}[\bar{z} z] \exp \left(-S_{\text {eff }}[\bar{z} z]\right)$ with the effective action

$$
S_{\mathrm{eff}}[\bar{z} z]=S_{\mathrm{BP}}[\bar{z} z]-\ln \operatorname{det}\left[\left(G^{\mathrm{MF}}\right)^{-1}+\delta G^{-1}(\bar{z} z)\right],
$$

where $S_{\mathrm{BP}}[\bar{z} z]=\int_{0}^{\beta} d \tau \int d^{3} r \bar{z} \partial_{\tau} z$ is the usual Berry's phase term. In Eq. (20), we have already split the total kernel $G^{-1}$ into a mean-field part $\left(G^{\mathrm{MF}}\right)^{-1}$ and a fluctuating part $\delta G^{-1}$,

$$
\begin{aligned}
\left(G^{\mathrm{MF}}\right)_{i j}^{-1} & =\left(\partial_{\tau}-\mu\right) \delta_{i j}+\left\langle i\left|H_{0}\right| j\right\rangle+N_{\mathrm{Mn}} J_{\mathrm{pd}} S s_{i j}^{z} \\
\delta G_{i j}^{-1}(\bar{z} z) & =\frac{J_{\mathrm{pd}}}{2}\left[\left(z s_{i j}^{-}+\bar{z} s_{i j}^{+}\right) \sqrt{2 N_{\mathrm{Mn}} S}-2 \bar{z} z s_{i j}^{z}\right]
\end{aligned}
$$

where $\mu$ denotes the chemical potential, and $i$ and $j$ range over a complete set of hole-band states (i.e., here, for the model with two parabolic bands, $i$ and $j$ label band wavevectors and spin, $\uparrow, \downarrow)$, and $s_{i j}^{z}$ and $s_{i j}^{ \pm}$are matrix elements of the itinerant-carrier spin matrices. The combination $\Delta=N_{\mathrm{Mn}} J_{\mathrm{pd}} S$ defines the mean-field energy to flip the spin of an itinerant carrier. The physics of the itinerant carriers is embedded in the effective action of the magnetic ions. It is responsible for the retarded and non-local character of the interactions between magnetic ions.

So far we have made no approximations. The independent spin-wave theory is obtained by expanding Eq. (20) up to quadratic order in $z$ and $\bar{z}$, i.e., spin excitations are treated as noninteracting HP bosons. This is a good approximation at low temperatures, where the number of spin excitations per Mn site is small.

We obtain (in the imaginary time Matsubara and coordinate Fourier representation) an action that is the sum of the temperature-dependent meanfield contribution and a fluctuation action. The latter is

$$
S_{\mathrm{eff}}[\bar{z} z]=\frac{1}{\beta V} \sum_{|\boldsymbol{k}| \leq k_{D}, m} \bar{z}\left(\boldsymbol{k}, \nu_{m}\right) D^{-1}\left(\boldsymbol{k}, \nu_{m}\right) z\left(\boldsymbol{k}, \nu_{m}\right) .
$$


A Debye cutoff $k_{D}$ with $k_{D}^{3}=6 \pi^{2} N_{\mathrm{Mn}}$ ensures that we include the correct number of magnetic-ion degrees of freedom, $|\boldsymbol{k}| \leq k_{D}$. The kernel of the quadratic action defines the inverse of the spin-wave propagator,

$$
D^{-1}\left(\boldsymbol{k}, \nu_{m}\right)=-i \nu_{m}+\frac{J_{\mathrm{pd}} p \xi}{2}+\frac{N_{\mathrm{Mn}} J_{\mathrm{pd}}^{2} S}{2 V} \sum_{\boldsymbol{q}} \frac{f\left[\epsilon_{\uparrow}(\boldsymbol{q})\right]-f\left[\epsilon_{\downarrow}(\boldsymbol{q}+\boldsymbol{k})\right]}{i \nu_{m}+\epsilon_{\uparrow}(\boldsymbol{q})-\epsilon_{\downarrow}(\boldsymbol{q}+\boldsymbol{k})}
$$

where $\xi=\left(p_{\downarrow}-p_{\uparrow}\right) / p$ is the fractional free-carrier spin polarization, and $\epsilon_{\uparrow, \downarrow}(\boldsymbol{q})$ is the energy of spin-up and spin-down valence-band holes, $\epsilon_{\uparrow, \downarrow}(\boldsymbol{q})=$ $\epsilon_{q} \pm \Delta / 2$, and $\epsilon_{q}=\hbar^{2} q^{2} /\left(2 m^{*}\right)$. The second term of Eq. (24) is the the energy for a Mn spin excitation in mean-field-theory, $\Omega^{\mathrm{MF}}=J_{\mathrm{pd}} p \xi / 2=x \Delta$. It differs from the itinerant-carrier spin splitting by the ratio of the spin densities $x=p \xi /\left(2 N_{\mathrm{Mn}} S\right)$, which is always much smaller than 1 in (III,Mn)V ferromagnets. Mean-field theory is, thus, recovered by dropping the last term in Eq. (24). It is this term that describes the response of the free-carrier system to changes in the magnetic-ion configuration.

\subsection{Elementary spin excitations}

We obtain the spectral density of the spin-fluctuation propagator by analytical continuation, $i \nu_{m} \rightarrow \Omega+i 0^{+}$and $A(\boldsymbol{k}, \Omega)=\operatorname{Im} D(\boldsymbol{k}, \Omega) / \pi$. In the following we consider the case of zero temperature, $T=0$. We find three different types of spin excitations [46].

Goldstone-mode spin waves. Our model has a gapless Goldstone-mode branch reflecting the spontaneous breaking of spin-rotational symmetry. The dispersion of this low-energy mode for four different valence-band carrier concentrations $p$ is shown in Fig. 9 (solid lines). At large momenta, $k \rightarrow \infty$, the spin-wave energy approaches the mean-field result $\Omega_{k}^{(1)} \rightarrow \Omega^{\mathrm{MF}}$ (shortdashed lines in Fig. 96). Expansion of the $T=0$ propagator for small momenta yields for the collective modes dispersion,

$$
\Omega_{k}^{(1)}=\frac{x / \xi}{1-x} \epsilon_{k}\left(\frac{3+2 \xi}{5}-\frac{4}{5} \xi \frac{\epsilon_{F}}{\Delta}\right)+\mathcal{O}\left(k^{4}\right),
$$

where $\epsilon_{F}$ is the Fermi energy of the majority-spin band. In strong and weakcoupling limits, $\Delta \gg \epsilon_{F}$ and $\Delta \ll \epsilon_{F}$, respectively, Eq. (25) simplifies to

$$
\begin{aligned}
\Omega_{k}^{(1)}=\frac{x}{1-x} \epsilon_{k}+\mathcal{O}\left(k^{4}\right) & \text { for } & \Delta \gg \epsilon_{F}, \\
\Omega_{k}^{(1)}=\frac{p}{32 N_{\mathrm{Mn}} S} \epsilon_{k}\left(\frac{\Delta}{\epsilon_{F}}\right)^{2}+\mathcal{O}\left(k^{4}\right) & \text { for } & \Delta \ll \epsilon_{F} .
\end{aligned}
$$

We note that the dependence of the spin-wave energy on the system parameters, namely the exchange interaction strength $J_{\text {pd }}$, hole concentration $p$, local-impurity density $N_{\mathrm{Mn}}$, and effective mass $m^{*}$ is different in these two 


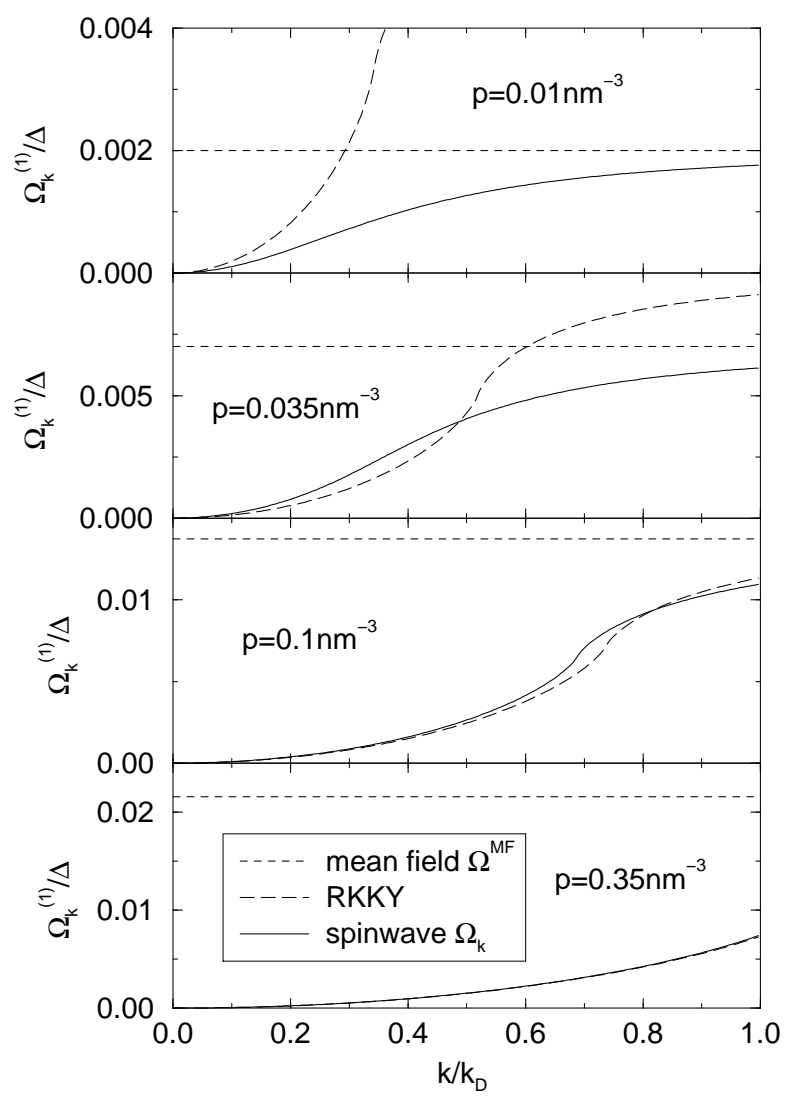

Fig. 9. Spin-wave dispersion (solid lines) for $J_{\mathrm{pd}}=0.06 \mathrm{eVnm}^{3}, m^{*}=0.5 m_{e}$, $N_{\mathrm{Mn}}=1 \mathrm{~nm}^{-3}$, and four different itinerant-carrier concentrations $p=0.01 \mathrm{~nm}^{-3}$, $0.035 \mathrm{~nm}^{-3}, 0.1 \mathrm{~nm}^{-3}$, and $0.35 \mathrm{~nm}^{-3}$. The ratio $\Delta / \epsilon_{F}$ is $2.79,1.21,0.67$, and 0.35 , which yields the fractional free-carrier spin polarization $\xi$ as $1,1,0.69$, and 0.31 . The short wavelength limit is the mean-field result $\Omega^{\mathrm{MF}}=x \Delta$ (short-dashed lines), and the long-dashed lines are the result obtained from an RKKY picture.

limits, indicating that the microscopic character of the gapless collective excitations differs qualitatively in the two limits. The energy of long-wavelength spin waves is determined by a competition between exchange and kinetic energies. To understand this in more detail one can impose the spin configuration of a static spin wave on the Mn spin system, evaluate the ground-state energy of the itinerant-carrier system in the presence of the generated exchange field, and compare this with the ground-state energy of a uniformly polarized state. The results of this calculation are explained briefly below; for details see Ref. 447. Given the Mn spin configuration, the valence-band carriers can either follow the spatial dependence of the Mn spin density in 
order to minimize the exchange energy, as they do in the strong-coupling limit $\Delta \gg \epsilon_{F}$, or minimize the kinetic energy by forming a state with a homogeneous spin polarization, as they do in the weak-coupling limit $\Delta \ll \epsilon_{F}$. The corresponding energy scales are provided by $\Delta$ and $\epsilon_{F}$, i.e., the crossover from one regime to the other is governed by the ratio $\Delta / \epsilon_{F}$.

Stoner continuum. We observe that the frequency $\nu_{m}$ is not only present in the first term of Eq. (24), it enters the third term, too. This is the reason why, in addition to the Goldstone mode, other spin excitations can appear in our model. They are absent in a static-limit description, i.e., when the frequency dependence of the third term of Eq. (24) is neglected.

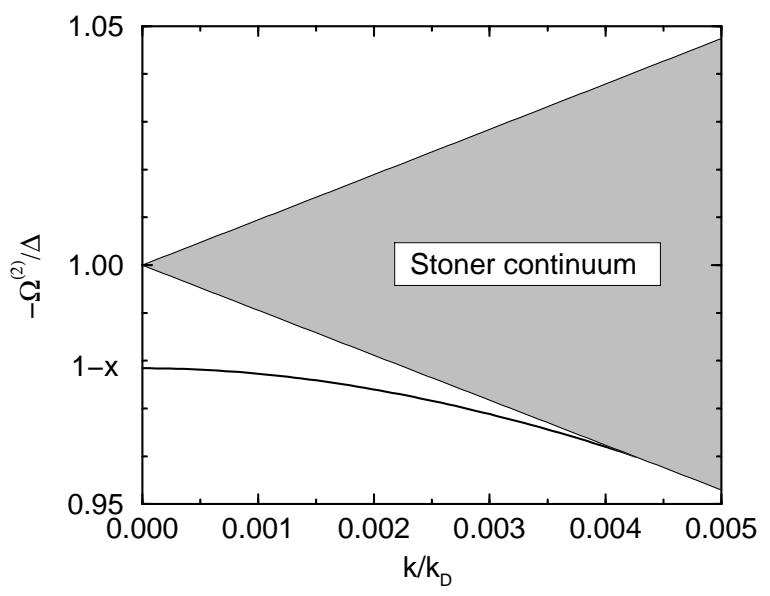

Fig. 10. Stoner excitations and optical spin-wave mode in the free-carrier system for $J_{\mathrm{pd}}=0.06 \mathrm{eVnm}^{3}, m^{*}=0.5 m_{e}, N_{\mathrm{Mn}}=1 \mathrm{~nm}^{-3}$, and $p=0.35 \mathrm{~nm}^{-3}$. In an RKKY picture these modes are absent.

We find a continuum of Stoner spin-flip particle-hole excitations. They correspond to flipping a single spin in the itinerant-carrier system and, since $x \ll 1$, occur in this simple model at much larger energies near the itinerantcarrier spin-splitting gap $\Delta$ (see Fig. 10). For $\Delta>\epsilon_{F}$ and zero temperature, all these excitations carry spin $S^{z}=+1$, i.e., increase the spin polarization. They therefore turn up at negative frequencies in the boson propagator we study. When $\Delta<\epsilon_{F}$, excitations with both $S^{z}=+1$ and $S^{z}=-1$ contribute to the spectral function. The continuum lies between the curves $-\Delta-\epsilon_{k} \pm$ $2 \sqrt{\epsilon_{k} \epsilon_{F}}$ and for $\Delta<\epsilon_{F}$ also between $-\Delta+\epsilon_{k} \pm 2 \sqrt{\epsilon_{k}\left(\epsilon_{F}-\Delta\right)}$. 
Optical spin waves. We find additional collective modes analogous to the optical spin waves in a ferrimagnet. Their dispersion lies below the Stoner continuum (see Fig. 10). At small momenta the dispersion is

$$
-\Omega_{k}^{(2)}=\Delta(1-x)-\frac{\epsilon_{k}}{1-x}\left(\frac{4 \epsilon_{F}}{5 x \Delta}-\frac{2-(2-5 x) / \xi}{5 x}\right)+\mathcal{O}\left(k^{4}\right) .
$$

The finite spectral weight at negative frequencies indicates that, because of quantum fluctuations, the ground state is not fully spin polarized.

\subsection{Comparison to RKKY and to the mean-field picture}

For comparison we evaluate the $T=0$ magnon dispersion assuming an RKKY interaction between magnetic ions. This approximation results from our theory if we neglect spin polarization in the itinerant carriers and evaluate the static limit of the resulting spin-wave propagator defined in Eq. 24). The Stoner excitations and optical spin waves shown in Fig. 10 are then not present and the Goldstone-mode dispersion is incorrect except when $\Delta \ll \epsilon_{F}$, as depicted in Fig. 9 (long-dashed lines).

In the mean-field picture, correlations among the Mn spins are neglected. The mean-field theory can be obtained in our approach by taking the Ising limit, i.e., replacing $\boldsymbol{S} \cdot s$ by $S^{z} s^{z}$. As mentioned before, this amounts to dropping the last term in Eq. (24). The energy of an impurity-spin excitation is then dispersionless, $\Omega^{\mathrm{MF}}=x \Delta$ (short-dashed line in Fig. 9), and always larger than the real spin-wave energy.

\subsection{Spin-wave dispersion for realistic bands}

For a quantitative analysis [50] of the spin-wave dispersion we extend our parabolic-band model to a six-band Kohn Luttinger Hamiltonian. The effective action for the HP bosons describing the Mn impurity spins is given by the same formal expression Eq. (20) with the contributions Eqs. (21) and (22) to the kernel. The difference is that for each Bloch wavevector $i$ and $j$ now label the states in a six-dimensional Hilbert space (instead of two dimensions for spin up and down), $H_{0}$ is the Kohn-Luttinger Hamiltonian, and $s_{i j}^{z}$ and $s_{i j}^{ \pm}$are $6 \times 6$ matrices.

The next step is again an expansion of the effective action up to quadratic order in $z$ and $\bar{z}$. In the two-band model, where spin is a good quantum number, only $\bar{z}\left(\boldsymbol{k}, \nu_{m}\right) z\left(\boldsymbol{k}, \nu_{m}\right)$ the combinations appear, see Eq. (23). Since the coherent state labels can can be viewed as boson creation and annihilation operators, these contributions are diagonal in total boson number. In the presence of spin-orbit coupling, however, spin is no longer a good quantum number, and the combinations $\bar{z}\left(\boldsymbol{k}, \nu_{m}\right) \bar{z}\left(-\boldsymbol{k},-\nu_{m}\right)$ and $z\left(\boldsymbol{k}, \nu_{m}\right) z\left(-\boldsymbol{k},-\nu_{m}\right)$ which increase or decrease the number of HP bosons, come into play.

Since our aim here is to derive the dispersion relations of the low-energy spin waves, rather than to address the full excitation spectrum including the 
Stoner continuum and the optical spin waves, we take the static limit as discussed in the context of the two-band model. After a Bogoliubov transformation, we obtain for the spin-wave energy

$$
\frac{\Omega_{\boldsymbol{k}}}{\Delta}=\frac{J_{\mathrm{pd}}}{2} \sqrt{\left(\frac{p \xi}{\Delta}-E_{\boldsymbol{k}}^{+-}\right)^{2}-\left|E_{\boldsymbol{k}}^{++}\right|^{2}}
$$

with the definition

$$
E_{\boldsymbol{k}}^{\sigma \sigma^{\prime}}=-\frac{1}{V} \sum_{\boldsymbol{q}} \sum_{\alpha \beta} \frac{f\left[\epsilon_{\alpha}(\boldsymbol{q})\right]-f\left[\epsilon_{\beta}(\boldsymbol{q}+\boldsymbol{k})\right]}{\epsilon_{\alpha}(\boldsymbol{q})-\epsilon_{\beta}(\boldsymbol{q}+\boldsymbol{k})} s_{\alpha \beta}^{\sigma} s_{\beta \alpha}^{\sigma^{\prime}}
$$

for $\sigma, \sigma^{\prime}= \pm$. The indices $\alpha$ and $\beta$ label the single-particle eigenstates for valence-band carriers at a given wavevector $\boldsymbol{q}$ and $\boldsymbol{q}+\boldsymbol{k}$, and $s_{\alpha \beta}^{ \pm}=\left\langle\alpha\left|s^{ \pm}\right| \beta\right\rangle$. The remaining task is to evaluate the fractional itinerant-carrier polarization $\xi$ and the quantities $E_{\boldsymbol{k}}^{+-}$and $E_{\boldsymbol{k}}^{++}$numerically.

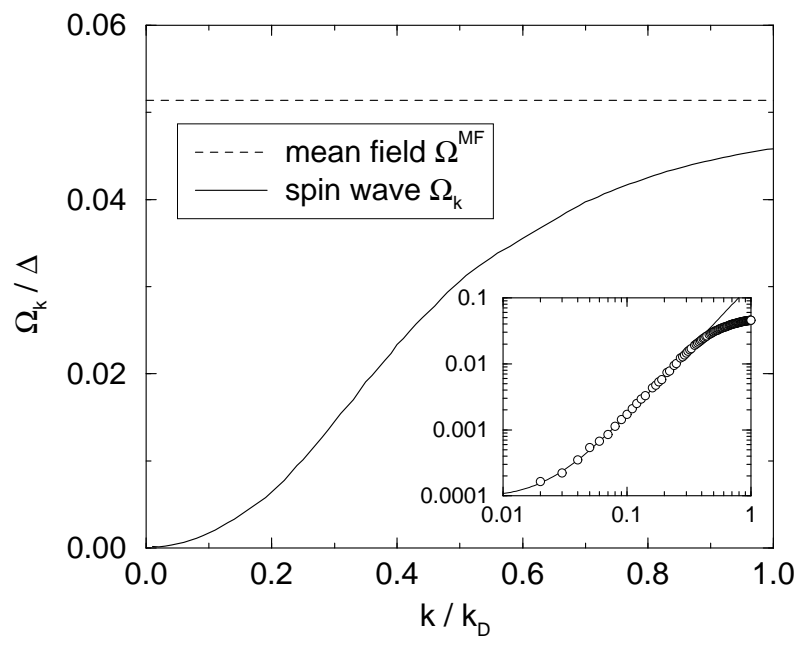

Fig. 11. Main panel: Spin-wave dispersion for the 6-band model for itinerant-carrier density $p=0.35 \mathrm{~nm}^{-3}$, impurity-spin concentration $N_{\mathrm{Mn}}=1.0 \mathrm{~nm}^{-3}$ and exchange coupling $J_{\mathrm{pd}}=0.068 \mathrm{eV} \mathrm{nm}^{-3}$. Inset: Spin-wave dispersion on a log-log plot (circles) and the parabolic fit (solid line).

In Fig. 11 we show the spin-wave dispersion for wavevectors $\boldsymbol{k}$ along the easy axis obtained using parameters valid for $(\mathrm{Ga}, \mathrm{Mn}) \mathrm{As}$ [5]. We observe that the effect of $E_{k}^{++}$in Eq. (29) is negligibly small and can, therefore, be dropped. Furthermore, we find that the dispersion is fairly independent of its wavevector direction, a property that is usually implicitly assumed in micromagnetic descriptions of magnetic materials. 
Spin stiffness. The quantized energy of a long-wavelength spin wave in a ferromagnet with uniaxial anisotropy can be written as

$$
\Omega_{k}=\frac{2 K}{N_{\mathrm{Mn}} S}+\frac{2 A}{N_{\mathrm{Mn}} S} k^{2}+\mathcal{O}\left(k^{4}\right),
$$

where $K$ is the anisotropy energy constant, and $A$ denotes the spin stiffness or exchange constant. While the anisotropy constant can be obtained from the mean-field energy for different magnetization orientations (see previous section), the virtue of the spin-wave calculation is to extract the spin stiffness as well.

In Fig. 12 we show the spin stiffness $A$ as a function of the itinerant-carrier density for two values of $J_{\mathrm{pd}}$ for both the isotropic two-band and the full sixband model. We find that the spin stiffness is much larger for the six-band calculation than for the two-band model. Furthermore, for the chosen range of itinerant-carrier densities the trend is different: in the two-band model the exchange constant decreases with increasing density, while for the six-band description we observe an increase with a subsequent saturation.

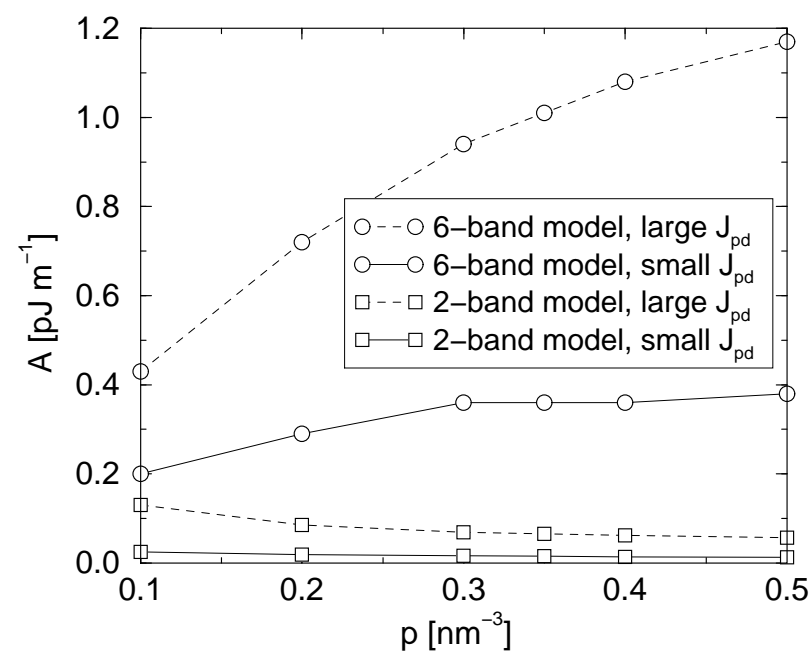

Fig. 12. Exchange constant $A$ as a function of itinerant-carrier density $p$ for the six-band and the two-band model for two different values of $J_{\mathrm{pd}}=0.068 \mathrm{eV} \mathrm{nm}^{-3}$ (solid lines) and $0.136 \mathrm{eV} \mathrm{nm}^{-3}$ (dashed lines). The impurity-spin concentration is chosen as $N_{\mathrm{Mn}}=1.0 \mathrm{~nm}^{-3}$, which yields $\Delta=0.17 \mathrm{eV}$ (solid lines) and $\Delta=0.34 \mathrm{eV}$ (dashed lines), respectively.

To understand this behavior we recall that the two-band model predicts a different dependence of $A$ on $p$ in the strong and weak-coupling limits with a crossover near $\Delta \sim \epsilon_{F}$, see Eqs. (26) and (27). The difference in the trends seen for the two- and six-band model in Fig. 12 is explained in part by the 
observation that, at given itinerant-carrier concentration $p$, the Fermi energy $\epsilon_{F}$ is much smaller when the six-band model is employed, where more bands are available for the carriers, than in the two-band case. Furthermore, we emphasize that, even in the limit of low carrier concentration, it is not only the (heavy-hole) mass of the lowest band which is important for the spin stiffness. Instead, a collective state in which the spins of the itinerant carriers follow the spatial variation of a Mn spin-wave configuration will involve the light-hole band, too. Our calculations show that accounting for the presence of this second more dispersive band is essential to understanding the success of mean-field theory. Crudely, the large mass heavy hole band dominates the spin-susceptibility and enables local magnetic order at high temperatures, while the dispersive light hole band dominates the spin stiffness and enables

long range magnetic order. The multi-band character of the semiconductor valence plays an essential role in the ferromagnetism of these materials.

\subsection{Limits on the Curie temperature}

Isotropic ferromagnets have spin-wave Goldstone collective modes whose energies vanish at long wavelengths,

$$
\Omega_{k}=D k^{2}+\mathcal{O}\left(k^{4}\right),
$$

where $k$ is the wavevector of the mode. Spin-orbit coupling breaks rotational symmetry which leads to a finite gap, see Eq. (31). According to our numerical studies, though, this gap is negligibly small as far as the suppression of ferromagnetism by collective spin excitations is concerned and can, therefore, be dropped for the present discussion. Each spin-wave excitation reduces the total spin of the ferromagnetic state by 1 . The coefficient $D=2 A /\left(N_{\mathrm{Mn}} S\right)$ is proportional to the spin stiffness $A$. These collective excitations are not accounted for in the mean-field approximation. If the spin stiffness is small, they will dominate the suppression of the magnetization at all finite temperatures and limit the critical temperature. In this case, the typical local valence-band carrier polarization remains finite above the critical temperature. Ferromagnetism disappears only because of the loss of long-range spatial coherence.

A rough upper bound on the critical temperature $T_{c}^{\text {coll }}$ can be obtained by the following argument which accounts for the role of collective fluctuations 48. The magnetization vanishes at the temperature where the number of excited spin waves equals the total spin of the ground state.

$$
N_{\mathrm{Mn}} S=\frac{1}{2 \pi^{2}} \int_{0}^{k_{D}} d k k^{2} n\left(\Omega_{k}\right),
$$

where $n\left(\Omega_{k}\right)$ is the Bose occupation number and the Debye cutoff, $k_{D}=$ $\left(6 \pi^{2} N_{\mathrm{Mn}}\right)^{1 / 3}$, ensures the correct number of magnetic ion degrees of free- 
dom. We therefore find that the critical temperature of a ferromagnet cannot exceed

$$
k_{B} T_{c}^{\mathrm{coll}}=\frac{2 S+1}{6} D k_{D}^{2}
$$

for $S \geq 5 / 2$ where $D$ is the $T=0$ spin-stiffness. To obtain this equation, we have assumed that the spin waves can be approximated as non-interacting Bose particles, replaced the dispersion by the long-wavelength limit Eq. (32), and noted that the critical temperature estimate is proportional to $D k_{D}^{2}$, justifying the use of the classical expression for the mode occupation number $n_{k} \approx k_{B} T / \Omega_{k}-1 / 2$. These considerations set an upper bound on the critical temperature which is proportional to the spin stiffness, a bound not respected by mean-field theory.

To get a qualitative but transparent picture we employ the two-band model with parabolic bands, and deduce the spin stiffness from Eqs. (26) and (27) for the strong and weak-coupling regime, respectively. For strong coupling, $\Delta / \epsilon_{F} \gg 1$, the exchange coupling completely polarizes the valenceband electrons, and we find (using $p \ll 2 N_{\mathrm{Mn}} S$ ) the $T_{c}$ bound

$$
T_{c}^{\mathrm{coll}, \mathrm{s}}=\frac{2 S+1}{12 S} \epsilon_{F}\left(\frac{p}{N_{\mathrm{Mn}}}\right)^{1 / 3} .
$$

For small $\Delta / \epsilon_{F}$, the weak-coupling or RKKY regime, exchange coupling is a weak perturbation on the band system. In this regime we get

$$
T_{c}^{\mathrm{coll}, \mathrm{RKKY}}=T_{c}^{\mathrm{MF}} \frac{2 S+1}{12(S+1) \sqrt[3]{2}}\left(\frac{N_{\mathrm{Mn}}}{p}\right)^{2 / 3}
$$

i.e., mean-field theory is reliable only for $p / N_{\mathrm{Mn}} \ll 1$, as expected since in this case the RKKY interaction has a range which is long compared to the distance between Mn spins.

We expect that the qualitative picture derived from the two-band model will persist for the six-band model. The actual values of the boundaries between the regimes indicated in Fig. 13 will, however, be shifted because of the important differences in the microscopic physics that determines the spin stiffness of the two models discussed above. We expect that the $T_{c}$ estimates derived in the preceding paragraph will be directly applicable to ntype carrier-mediated ferromagnets. As a consequence we expect that it will be impossible to achieve large ferromagnetic transition temperatures in n-type semiconductors with carrier mediated ferromagnetism. The $\sim 5 \%$ reduction of (Ga,Mn)As mean-field $T_{c}$ due to spin fluctuations, mentioned in Section 4.1, was obtained using the six-band model and solving self-consistently Eq. (34) and $D\left(T_{c}^{\text {coll }}\right)=D(T=0)\langle S\rangle\left(T_{c}^{\text {coll }}\right) / S$. Larger reductions compared to meanfield-theory estimates are expected in some hosts, but mean-field-theory retains a qualitative validity. 


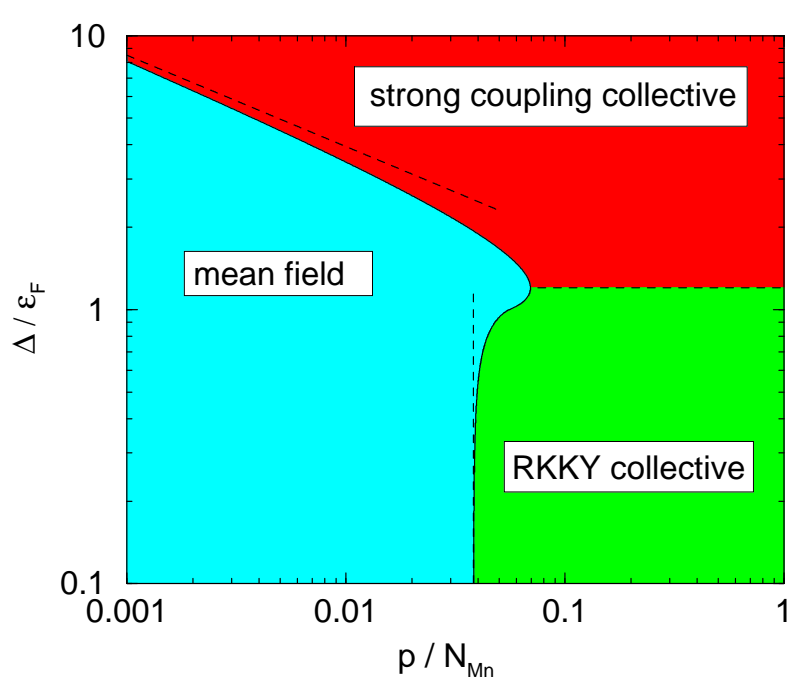

Fig. 13. Critical-temperature-limit regimes for the two-band model. In the meanfield regime $T_{c}$ is limited by individual $\mathrm{Mn}$ spin fluctuations. In the collective regimes, the critical temperature is limited by long-wavelength fluctuations with a stiffness proportional to the bandwidth for weak (RKKY) exchange coupling and inversely proportional to the bandwidth for strong exchange coupling. At the solid line $T_{c}^{\mathrm{MF}}=T_{c}^{\mathrm{coll}}$. Dashed lines: expansions for large and small $\Delta / \epsilon_{F}$, Eqs. (35) and (36), and the crossover from the RKKY to the strong coupling collective regime.

\section{Collective fluctuations beyond spin wave theory and continuum approximation}

In the preceding sections we described the magnetic properties of Mn-doped semiconductors by a model of itinerant carriers which are exchange-coupled to localized magnetic moments formed by the dopants. An important property, resulting from the growth process of such materials, is that the Mn acceptors are distributed at random on the cation sites of the underlying crystal lattice. To this point in this chapter, we have used a continuum approximation for the Mn ion distribution that yields a disorder-free problem. The continuum approximation makes it possible to obtain some results analytically, and also crucially simplifies numerical calculations. However, it neglects substitutional disorder in the Mn positions which can have a substantial impact on the ferromagnetism. That this is true is evident from the fact that magnetic properties of presently available samples are often sensitive to the conditions of their fabrication, and reproducibility is achieved only if the growth parameters are carefully controlled [5]. Moreover, recent studies of post-growth annealed $(\mathrm{Ga}, \mathrm{Mn})$ As samples have revealed that the magnetic [52] as well as the structural [53] properties can depend crucially on the type of defects and disorder present in the system. 
The spin-wave theory presented in the previous section describes collective excitations of the ion spin system (modeled as a continuum) in terms of Gaussian fluctuations around the ferromagnetic ground state in the manybody path integral. This non-interacting spin-wave theory is exact at low temperatures, where deviations from the ordered ground state are small, but is less reliable when the temperature is raised toward the ferromagnetic transition temperature.

In this section we complement the theoretical approaches described above by methods which (i) take disorder effects due to the randomly chosen ion positions into account and (ii) are able to address directly the region of larger deviations of the spin configuration from the ferromagnetically ordered state. Specifically, we present results of Monte Carlo simulations [48,49] which treat the minimal model without approximation. Finally we report on a rigorous stability analysis of the perfectly ferromagnetically ordered collinear state of $\mathrm{Mn}$ ions in the presence of disorder [54]. We predict that noncollinear ferromagnetism is common in $(I I I, M n) V$ semiconductors; the robust collinear ferromagnetic states that have the highest ferromagnetic transition temperatures occur only when the carriers are relatively weakly localized around individual Mn ions.

\subsection{Model considerations}

The considerations in this section are based on the kinetic-exchange model discussed in the previous sections. To account for the finite extent of the Mn ions 28] in the exchange term we replace $J_{\mathrm{pd}} \boldsymbol{s}\left(\boldsymbol{R}_{I}\right) \cdot \boldsymbol{S}_{I}$ by $\int d^{3} r J(\boldsymbol{r}-$ $\left.\boldsymbol{R}_{I}\right) \boldsymbol{s}(\boldsymbol{r}) \cdot \boldsymbol{S}_{I}$ with the finite-range exchange parameter

$$
J(\boldsymbol{r})=\frac{J_{\mathrm{pd}}}{\left(2 \pi a_{0}^{2}\right)^{\frac{3}{2}}} e^{-r^{2} /\left(2 a_{0}^{2}\right)} .
$$

Both the strength $J_{\mathrm{pd}}$ and range $a_{0}$ of this interaction are phenomenological parameters to be fixed by comparison with experiment or, ideally, to be extracted from first principles electronic structure calculations. Note that the exchange-coupling range parameter $a_{0}$ in Eq. (37) is required in our calculations once the discreteness of the Mn ions is acknowledged; exchangecoupling shifts of quasiparticle energies would diverge otherwise. Given this finite range, the only approximation we make below in treating the minimal model is that we treat the Mn spins classically. Because of the relatively large value of the $\mathrm{Mn}$ ion spins, this approximation should have minimal consequence except for the leading low temperature magnetization suppression.

We are interested in thermal expectation values of the form

$$
\bar{f}=\frac{1}{\mathcal{Z}} \int_{0}^{2 \pi} d \varphi \int_{0}^{\pi} d \vartheta \sin \vartheta \operatorname{Tr}\left\{\hat{f}(\vartheta, \varphi) e^{-\beta \mathcal{H}}\right\},
$$

where $\beta$ is the inverse temperature, $\mathcal{Z}$ the partition function, and $\vartheta, \varphi$ are shorthand notations for the whole set of classical spin coordinates. The quantity $\hat{f}(\vartheta, \varphi)$ is a function of the ion spin angles and an operator with respect 
to the quantum mechanical carrier degrees of freedom over which the trace is performed. In practice we replace the fermion trace by a ground state expectation value, since the temperatures of interest will always be much smaller than the Fermi energy. For typical carrier densities $p$ of order $0.1 \mathrm{~nm}^{-3}$, the Fermi temperature for the carriers is typically larger than $1000 \mathrm{~K}$, compared to ferromagnetic critical temperatures $\sim 100 \mathrm{~K}$. Thermal effects in the carrier system are therefore negligible. Thus,

$$
\bar{f}=\frac{1}{\mathcal{Z}} \int_{0}^{2 \pi} d \varphi \int_{0}^{\pi} d \vartheta \sin \vartheta\langle 0|\hat{f}(\vartheta, \varphi)| 0\rangle e^{-\beta\langle 0|\mathcal{H}| 0\rangle},
$$

where $|0\rangle$ denotes the groundstate of non-interacting fermions with the appropriate band Hamiltonian and a Zeeman-coupling term $h$ whose effective magnetic field $H_{\text {eff }}$ is due to exchange interactions with the localized spins,

$$
\begin{aligned}
h & =g \mu_{B} \int d^{3} r \boldsymbol{s}(\boldsymbol{r}) \cdot \boldsymbol{H}_{\mathrm{eff}}(\boldsymbol{r}) \\
\boldsymbol{H}_{\mathrm{eff}}(\boldsymbol{r}) & =\sum_{I} J\left(\boldsymbol{r}-\boldsymbol{R}_{I}\right) S \hat{\Omega}_{I} /\left(g \mu_{B}\right)
\end{aligned}
$$

where $\hat{\Omega}_{I}=\left(\sin \theta_{I} \cos \phi_{I}, \sin \theta_{I} \sin \phi_{I}, \cos \theta_{I}\right)$ is the direction of the classical spin at $\boldsymbol{R}_{I}$. In the following we denote thermal expectation values of quantities defined in terms of classical spin orientation variables by $\langle\cdot\rangle$ and quantum mechanical expectation values within the carriers ground state by $\langle 0|\cdot| 0\rangle$.

\subsection{Remarks on the Monte Carlo method}

A standard way to evaluate expectation values of the form Eq. (39) is to use classical Monte Carlo algorithms which perform a random walk in phase space of the classical variables $(\vartheta, \varphi)$. The probabilities governing this Monte Carlo dynamics are specified by the dependence of many-fermion energy on the localized-spin configuration. The many-fermion ground state is a Slater determinant whose single-particle orbitals are the lowest energy eigenstates of a single-band or multi-band Hamiltonian. For the case of a parabolic band, the matrix elements of the corresponding one-particle Hamiltonian in a planewave basis read

$$
\left\langle\boldsymbol{k}^{\prime} \sigma^{\prime}|\mathcal{H}| \boldsymbol{k} \sigma\right\rangle=\frac{\hbar^{2} k^{2}}{2 m^{*}} \delta_{\boldsymbol{k}^{\prime} \boldsymbol{k}} \delta_{\sigma^{\prime} \sigma}+\frac{S}{2 L^{3}} \sum_{I} J_{\boldsymbol{k}-\boldsymbol{k}^{\prime}} e^{i\left(\boldsymbol{k}-\boldsymbol{k}^{\prime}\right) \boldsymbol{R}_{I}} \hat{\Omega}_{I} \cdot \boldsymbol{\tau}_{\sigma^{\prime} \sigma}
$$

where $\boldsymbol{k}$ and $\sigma$ denote wavevector and spin indices, respectively, $J_{\boldsymbol{k}}$ is the Fourier transform of $J(\boldsymbol{r})$, and $L$ the edge length of the simulation cube. Periodic boundary conditions restrict the admissible values of wavevector components to integer multiples of $2 \pi / L$. In Eq. 41, $\boldsymbol{\tau}$ is the vector of Pauli spin matrices.

Since the many-particle ground state of the carrier system has to be computed at each Monte Carlo step, the computational effort required for the 
present calculations is much larger than in simple classical spin models. In the usual Metropolis algorithm, a single spin orientation is altered at each step. If this algorithm were employed here, the time required to diagonalize the single-particle Hamiltonian each time would severely limit the efficiency of the algorithm. We therefore use the Hybrid Monte Carlo algorithm, which was introduced in the mid 1980's in the context of lattice field theories [55]. In this method all classical variables are altered in one Monte Carlo step. This drastically reduces the number of matrix diagonalizations required to explore statistically important magnetic configurations. The Hybrid algorithm is a powerful method for Monte Carlo simulations in systems containing coupled classical and quantum mechanical degrees of freedom.

A concrete Monte Carlo simulation necessarily works in a system of finite size. In the present case, calculation of the fermion ground state also requires truncation of the plane-wave expansion we use for the independent particle wavefunctions that diagonalize the Hamiltonian (41). We are able to obtain convergence with respect to this truncation only when the range of the exchange interaction $a_{0}$ is not too short. taking into account a finite number of plane-wave states entering the single-particle carrier Hamiltonian (41). The importance of these finite-size effects in real and reciprocal space (using periodic boundary conditions), and their interplay with the regularization parameter $a_{0}$, are discussed in detail in Ref. [49]. In the following we shall leave aside such technical aspects and concentrate on the physical results.

\subsection{Numerical Monte Carlo Results}

In this subsection we present numerical Monte Carlo results. We concentrate on the spin polarizations of the Mn ions and the carriers as a function of temperature and address the ferromagnetic transition. We start with the two-band model.

Two-band model. Fig. 14 shows typical magnetization data as a function of temperature. These results were obtained for a Mn ion density of $N_{\mathrm{Mn}}=$ $1.0 \mathrm{~nm}^{-3}$, a carrier density $p=0.1 \mathrm{~nm}^{-3}$ in a cubic simulation volume of $V=540 \mathrm{~nm}^{3}$, i.e., the system contains $540 \mathrm{Mn}$ ions and 54 carriers. The effective band mass is half the bare electron mass, and the chosen exchange parameter is $J_{\mathrm{pd}}=0.15 \mathrm{eVnm}^{3}$. Here and in the following Monte Carlo data the range parameter for the exchange coupling is $a_{0}=0.1 \mathrm{~nm}$.

The main panel shows the average polarization of the Mn spins,

$$
M=\frac{1}{N_{\mathrm{Mn}} V}\left\langle\left|\boldsymbol{S}_{\mathrm{tot}}\right|\right\rangle
$$


i. e. the thermally averaged modulus of the total ion spin, along with the carrier magnetization,

$$
m=\frac{1}{p V}\left\langle\left|\left\langle 0\left|s_{\text {tot }}\right| 0\right\rangle\right|\right\rangle,
$$

which is the ensemble average of the modulus of the total ground-state carrier spin. Both quantities are divided by the number of particles and are close to their maximum values at low temperatures. At higher temperatures they show the expected transition to a paramagnetic phase. The critical temperature of this ferromagnetic transition is most readily estimated from numerical results for the magnetization fluctuations:

$$
\begin{aligned}
g_{\mathrm{Mn}} & =\frac{1}{N_{\mathrm{Mn}} V}\left(\left\langle\left|\boldsymbol{S}_{\mathrm{tot}}\right|^{2}\right\rangle-\left\langle\left|\boldsymbol{S}_{\mathrm{tot}}\right|\right\rangle^{2}\right), \\
g_{p} & =\frac{1}{p V}\left(\left\langle\left|\left\langle 0\left|\boldsymbol{s}_{\mathrm{tot}}\right| 0\right\rangle\right|^{2}\right\rangle-\left\langle\left|\left\langle 0\left|\boldsymbol{s}_{\mathrm{tot}}\right| 0\right\rangle\right|\right\rangle^{2}\right) .
\end{aligned}
$$

These two fluctuations per particle are plotted in the insets of Fig. 14.

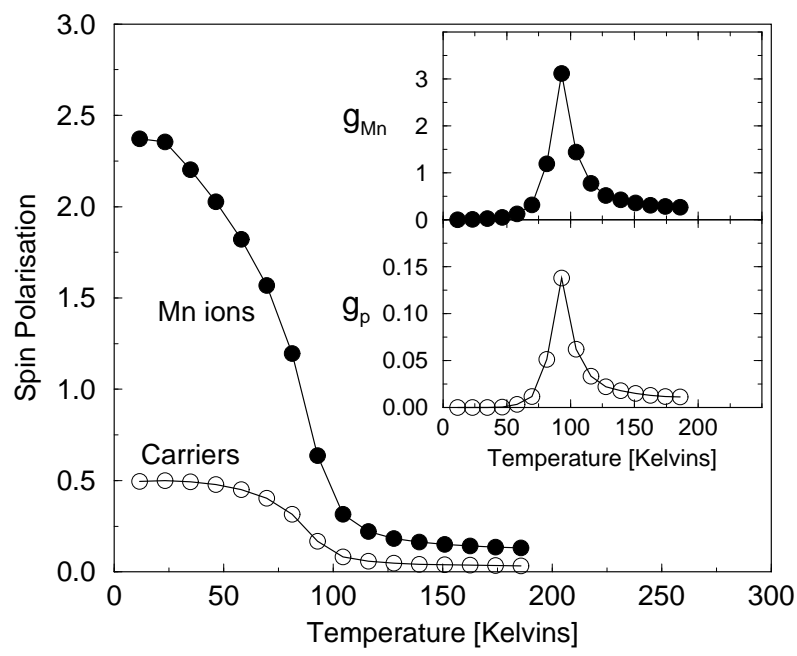

Fig. 14. Magnetization curves for Mn ions and carriers. The upper and lower inset show the magnetic fluctuations for the Mn ions and the carriers, respectively. Both differ by a factor of approximately 25 reflecting the square of the ratio of spin lengths. The density of $\mathrm{Mn}$ ions is $N_{\mathrm{Mn}}=1.0 \mathrm{~nm}^{-3}$, the carrier density is $p=$ $0.1 \mathrm{~nm}^{-3}$ in a cubic volume of $V=540 \mathrm{~nm}^{3}$. The band mass is half the bare electron mass with an exchange parameter of $J_{\mathrm{pd}}=0.15 \mathrm{eVnm}^{3}$.

They both show a pronounced peak at a temperature $T \sim 100 \mathrm{~K}$, defining the finite-system transition temperature for these model parameter values. In fact, in a region around this transition the two data sets differ by a factor 
of approximately 25 , which is the square of the ratio of the two spin lengths entering the expressions (44) and (45), respectively. This observation shows explicitly that the correlation length is the same for $\mathrm{Mn}$ ions and the carrier system near the transition, that is both approach the finite system size of the simulation.

Our Monte Carlo approach clearly reproduces the expected ferromagnetic transition. Ferromagnetism still occurs when randomness in the Mn positions is accounted for. The transition temperature $T_{c}$ can be determined unambiguously and consistently from the positions of very pronounced peaks in total magnetization fluctuations of both the Mn ions and the carriers.

Results for $\boldsymbol{T}_{\boldsymbol{c}}$. We now turn to the transition temperature $T_{c}$ for the two-band model. Within mean-field theory this quantity is given by Eq. (6). Our objective here is not to make a quantitative prediction of the critical temperature for particular ferromagnetic semiconductor systems. By doing a numerically exact calculation for a model that captures much of the physics, however, we hope to shed light on the range of validity and the sense and magnitude of likely corrections to mean-field-theory $T_{c}$ estimates.

The mean-field expression for $T_{c}$ can be obtained by averaging the ion-spin and carrier polarizations over space. The effective field which each Mn spin experiences due to a finite carrier polarization is then constant in space and the carrier bands are in turn rigidly spin split by $\Delta=J_{\mathrm{pd}} N_{\mathrm{Mn}} S$. (The limit in which mean-field theory is exact can be achieved in our model by letting $a_{0} \rightarrow \infty$ in Eq. (37).) Mean-field theory, which neglects spatial fluctuations and correlations between carriers and Mn spins, predicts that $T_{c}$ is quadratic in the exchange parameter $J_{\mathrm{pd}}$ and linear in the effective band mass $m^{*}$, when correlations in the itinerant system are neglected.

Here we determine the critical temperature $T_{c}$ with the help of our Monte Carlo scheme. In Fig. 15 we show results for Mn densities $N_{\mathrm{Mn}}=1.0 \mathrm{~nm}^{-3}$ and a mean-field band splitting $\Delta=J_{\mathrm{pd}} N_{\mathrm{Mn}} S=0.5 \mathrm{eV}$. The left panel shows the dependence of the critical temperature on the carrier effective mass. This dependence is very important for the search for diluted magnetic semiconductor systems with $T_{c}$ 's larger than room temperature. In the meanfield approximation, $T_{c}$ grows linear with increasing mass. The Monte Carlo results clearly deviate from this prediction suggesting a saturation of $T_{c}$ at carrier masses close to the bare electron mass. For even higher masses, we expect the electrons to behave more classically and localize around individual Mn spins, suppressing long-range order further. In this limit the electronic energy cost of changes in the relative orientations of nearby Mn spins will get smaller causing $T_{c}$ to decline, and eventually ferromagnetism will disappear. This is consistent with the observation that, within the continuum model, the spin stiffness declines as $1 / m^{*}$ for large band masses 46, 47, 48, As mentioned earlier, the spin stiffness tends to be remain substantially larger when coupled light and heavy holes are retained in the calculation. Still, this calculation 


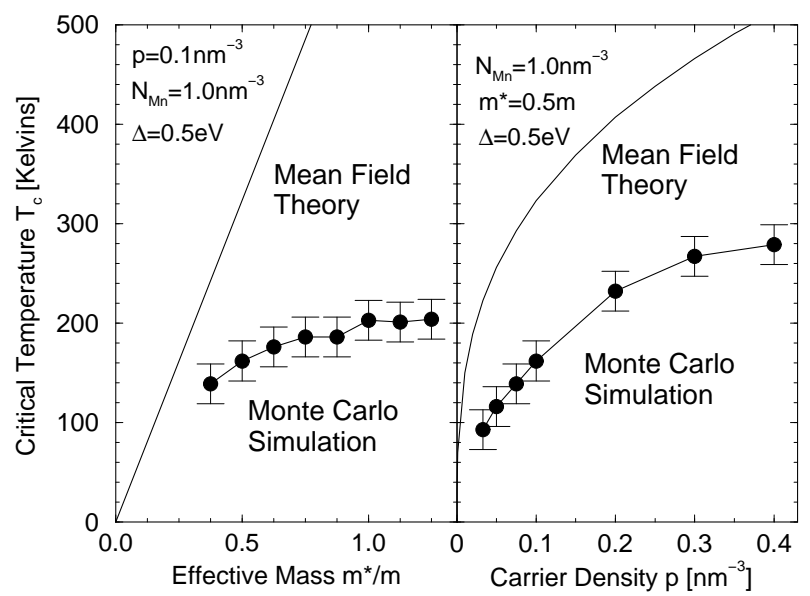

Fig. 15. The critical temperature $T_{c}$ as a function of the carrier mass (left panel) and the carrier density (right panel). The exchange parameter is in both cases $J_{\mathrm{pd}}=0.2 \mathrm{eVnm}^{3}$ leading to a zero temperature mean-field spin splitting of $\Delta=$ $J_{\mathrm{pd}} N_{\mathrm{Mn}} S=0.5 \mathrm{eV}$. The results of the Monte Carlo runs are compared with the mean-field predictions.

demonstrates that mean-field-theory must be regarded with some caution and its validity must be checked in each new circumstance.

To discuss the critical temperature as a function of the exchange coupling parameter $J_{\text {pd }}$, we observe that the Hamiltonian of itinerant carriers satisfies the scaling relation

$$
\beta \mathcal{H}\left(m^{*}, J_{\mathrm{pd}}\right)=\frac{\beta}{q} \mathcal{H}\left(\frac{m^{*}}{q}, q J_{\mathrm{pd}}\right)
$$

with $q>0$. Therefore the saturation of $T_{c}$ as a function of the effective mass at fixed $J_{\mathrm{pd}}$ corresponds to a linear dependence of $T_{c}$ on $J_{\mathrm{pd}}$ at fixed $m^{*}$. This contrasts with the mean-field prediction $T_{c}^{M F} \propto J_{\mathrm{pd}}^{2}$.

In the right panel of Fig. 15 we show $T_{c}$ as a function of the carrier density. Here the Monte Carlo approach also clearly yields a lower critical temperatures than mean-field theory. For still higher carrier densities the typical distance between nearby Mn ions will become larger than the band electron Fermi wavelength, causing the sign of the typical exchange coupling to oscillate in an RKKY fashion. As we shall see in the next subsection, the resulting frustration makes the ferromagnetic state unstable, possibly leading to a regime of spin-glass order. Disordered states can also occur when the exchange coupling becomes strong.

Six-band model. We now turn to the six-band model in which the kinetic energy part is given by the Kohn-Luttinger model. Fig. 16 shows typ- 
ical magnetization data for the (Ga,Mn)As system, assuming exchange coupling $J_{\mathrm{pd}}=0.15 \mathrm{eVnm}^{3}$, carrier density is $p=0.1 \mathrm{~nm}^{-3}$, and Mn ion density $N_{\mathrm{Mn}}=1.0 \mathrm{~nm}^{-3}$ in a volume of $V=280 \mathrm{~nm}^{3}$. As in the case of parabolic

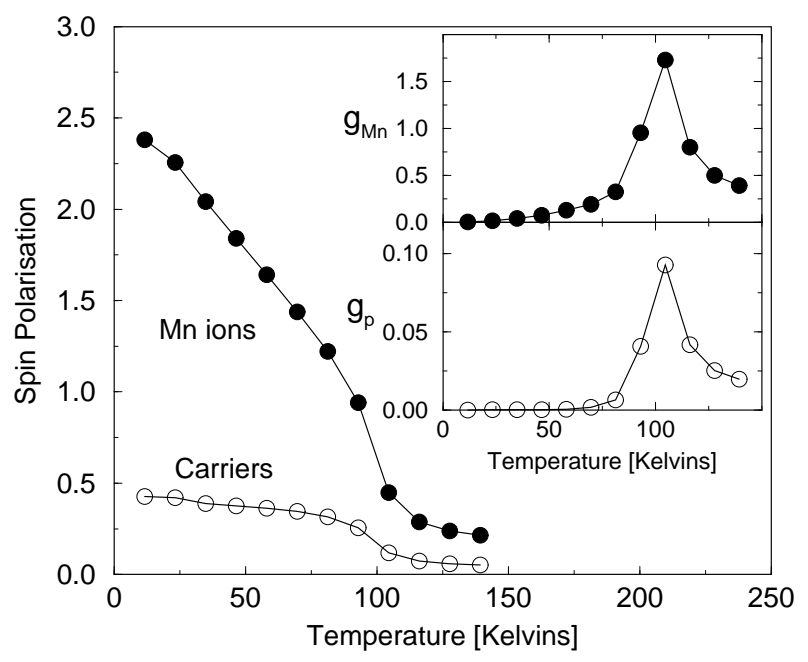

Fig. 16. Magnetization curves for $\mathrm{Mn}$ ions and carriers in the six-band model for n exchange coupling of $J_{\mathrm{pd}}=0.15 \mathrm{eVnm}^{3}$. The carrier density is $p=0.1 \mathrm{~nm}^{-3}$ with an $\mathrm{Mn}$ ion density of $N_{\mathrm{Mn}}=1.0 \mathrm{~nm}^{-3}$ in a volume of $V=280 \mathrm{~nm}^{3}$. As in the case of parabolic bands, the ferromagnetic transition in clearly and consistently signaled by pronounced peaks in the magnetic fluctuations shown in the insets.

bands, a ferromagnetic transition is clearly signaled by pronounced peaks in the magnetic fluctuations of both $\mathrm{Mn}$ ions and carriers. We find that, in contrast to the parabolic two-band model, the carrier magnetization is already reduced at temperatures well below $T_{c}$. In fact, because of strong spin-orbit coupling in the valence band, full polarization of the carrier spins never occurs. Another difference compared to the parabolic-band model concerns the shape of the magnetic fluctuations for the $\mathrm{Mn}$ ions and the carriers as a function of temperature. Although both curves indicate the same value for $T_{c}$ for these parameter values, their shape in the vicinity of $T_{c}$ is slightly different and the ratio of their fluctuations is smaller than 25 (the square of the ratio of spin lengths involved). These differences arise because of the more complicated band structure and the spin-orbit coupling present in the Kohn-Luttinger Hamiltonian. In the right panel of Fig. 17 we plot the transition temperature as a function of the exchange coupling $J_{\mathrm{pd}}$ for two different system sizes. Both data sets agree within error bars and show a linear dependence of $T_{c}$ on $J_{\text {pd }}$. This finding is the same as for the two-band model and contrasts with mean-field theory which predicts $T_{c} \propto J_{\text {pd }}^{2}$. The left panel of Fig. 17 shows the transition temperature as a function of $J_{\mathrm{pd}}$ for the parabolic 


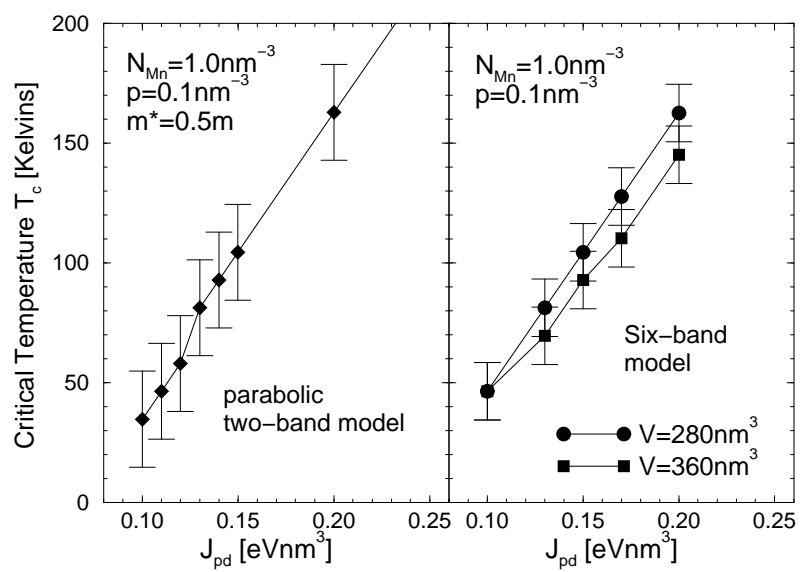

Fig. 17. The right panel shows critical temperature $T_{c}$ as a function of the exchange parameter $J_{\mathrm{pd}}$ for the same particle densities as in Fig. 16 and two different system sizes. Both data sets agree within error bars and show a linear dependence of $T_{c}$ on $J_{\mathrm{pd}}$. In the left panel, the corresponding data for the parabolic two-band model with an effective mass of half the bare electron mass is plotted. The latter system is a reasonable approximation to the six-band case.

model for an effective mass $m^{*}=0.5 m_{e}$. This value is close to the heavy-hole mass in the Kohn-Luttinger model for parameters appropriate for GaAs. The data in the left panel can be obtained from the left panel of Fig. 15 via the scaling relation (46). Comparing the two panels of Fig. 17 demonstrates that, in the range of carrier densities studied here, a single parabolic band with an effective mass close to that of the heavy-hole Kohn-Luttinger-model band provides a reasonably good approximation to the behavior of the six-band system. We expect larger differences to occur in the strong coupling regime, which presents technical difficulties to the Monte Carlo calculations.

\subsection{Disorder effects and noncollinear ferromagnetism}

The magnetization data of Fig. 14 were obtained by averaging over five different realizations of the $\mathrm{Mn}$ ions. In fact over the range of parameters we have explored, except for the larger values of $p / N_{\mathrm{Mn}}$, results for different disorder realizations differ only very weakly from each other. This is illustrated in Fig. 18, where the magnetization curves underlying the averaged results of Fig. 14 are plotted. Those five datasets are hardly distinguishable from each other. Positional disorder in the localized magnetic moments of $\mathrm{Mn}$ ions can however affect the nature of the ground state itself in some circumstances. As seen from Fig. 14, the ion spin magnetization seems to saturate at low temperatures at a value slightly smaller than its maximum, given by the Mn spin length of 5/2. Moreover, as shown in Fig. 18, this behavior occurs 


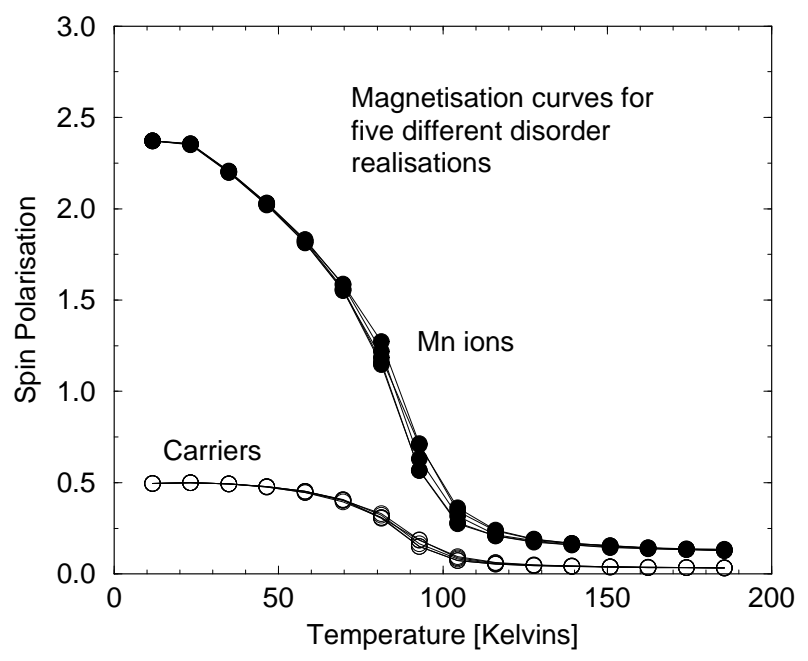

Fig. 18. Magnetization curves for five different realizations of Mn positions underlying the averaged data of Fig. 14.

quite consistently for different disorder realizations. As we see below, the effect is due to the randomness in Mn positions which, combined with the RKKY-like oscillations in the effective coupling between Mn spins, makes the perfectly ferromagnetically ordered collinear state state unstable and leads to noncollinear ferromagnetism.

We now outline a theory of magnetic fluctuations around a given state within a path integral formalism similar to the spin-wave approach presented in Section 5.2 for fluctuations around the collinear ferromagnetic state. In contrast to the spin-wave calculations, here we do not approximate the Mn magnetic moments by a continuum but retain them, as in the Monte Carlo approach, as individual spins of length $S=5 / 2$ placed at arbitrary locations. Let us first consider magnetic fluctuations around the perfectly ferromagnetically ordered collinear state having all Mn spins oriented in parallel.

Repeating the steps described in Section 5, but keeping the Mn spins as individual objects, one obtains the following expression for the fluctuation part of the effective action in up to second order in the bosonic spin variables:

$$
\mathcal{S}_{\mathrm{eff}}=\frac{1}{\beta} \sum_{m} \sum_{I, J} \bar{z}_{I}\left(\nu_{m}\right) D_{I J}^{-1}\left(\nu_{m}\right) z_{J}\left(\nu_{m}\right)
$$

where $\nu_{m}=2 \pi m / \beta$ is a Matsubara frequency. $z_{I}\left(\nu_{m}\right)$ stands for the bosonic Holstein-Primakoff field of Mn spin $I$ that describes deviations from a fully aligned state. The fluctuation matrix $D_{I J}^{-1}\left(\nu_{m}\right)$ reads

$$
D_{I J}^{-1}\left(\nu_{m}\right)=L_{I J}\left(\nu_{m}\right)+K_{I J}\left(\nu_{m}\right)
$$


with

$$
\begin{aligned}
L_{I J} & =\delta_{I J}\left(-i \nu_{m}-\int d^{3} r J\left(\boldsymbol{r}-\boldsymbol{R}_{I}\right)\left\langle s^{z}(\boldsymbol{r})\right\rangle\right), \\
K_{I J} & =\frac{S}{2} \sum_{\alpha, \beta}\left[\frac{f\left(\eta_{\alpha}\right)-f\left(\eta_{\beta}\right)}{i \nu_{m}+\eta_{\alpha}-\eta_{\beta}} F_{I}^{\alpha \downarrow, \beta \uparrow} F_{J}^{\beta \uparrow, \alpha \downarrow}\right] .
\end{aligned}
$$

Here $\langle\boldsymbol{s}(\boldsymbol{r})\rangle$ is the expectation value of the carrier spin density, $f$ the Fermi function, and

$$
F_{I}^{\alpha \sigma, \beta \mu}=\int d^{3} r J\left(\boldsymbol{r}-\boldsymbol{R}_{I}\right) \bar{\psi}_{\alpha \sigma}(\boldsymbol{r}) \psi_{\beta \mu}(\boldsymbol{r})
$$

with $\psi_{\alpha \sigma}(\boldsymbol{r})$ being the spin component $\sigma$ of the carrier wave function with label $\alpha$ and energy $\varepsilon_{\alpha}=\eta_{\alpha}+\mu$, where $\mu$ is the chemical potential for the carriers All quantities referring to the carrier system are to be evaluated for the collinear orientation of Mn spins.

The zero-frequency $(m=0)$ contribution to the effective action (47) describes the energy of static fluctuations around the collinear state. For this state to be stable, the matrix $D_{I J}^{-1}(0)$ must have non-negative eigenvalues only, while the occurrence of negative eigenvalues of this matrix indicates that the perfectly collinear state is not the ground state. This interpretation of the zero-frequency fluctuation term is confirmed by the observation that this contribution is also obtained (at zero temperature) by a standard perturbation theory for the carrier ground state energy with respect to small deviations of the ion spins from collinear orientation. The formalism given above embeds this finding in a more general theory of dynamic fluctuations at finite temperature. However, we shall concentrate in the following on static ground state properties, i.e. on $T=0$, where the Fermi functions become step functions.

We note that for any arrangement of the Mn positions $R_{I}$, the matrix $D_{I J}^{-1}(0)$ contains a zero eigenvalue corresponding to a uniform rotation of all spins. The eigenvalues of $D_{I J}^{-1}(0)$ are proportional to magnetic excitation energies. In this sense the eigenvalue distribution of $D_{I J}^{-1}(0)$ can be interpreted as a density of states for magnetic excitations.

We have evaluated the spectrum of $D_{I J}^{-1}(0)$ in systems given by a simulation cube with periodic boundary conditions averaging over different realizations of the Mn positions. The single-particle wavefunctions $\psi_{\alpha \sigma}(\boldsymbol{r})$ are computed in a plane-wave basis taking into account wavevectors $\boldsymbol{q}$ with length up to an appropriate cutoff $q_{c}$. The same truncated plane-wave basis is used to compute the quantities (51) entering (50). Note that, for fluctuations around the collinear ferromagnetic state, $D_{I J}^{-1}(i \omega)$ is always real and symmetric for real $\omega$ since all carrier wavefunctions have, for a given spin projection $\sigma$, the same coordinate-independent phase. This follows from the fact that the single-particle Hamiltonian describes for each spin projection just the problem of a spinless particle in a potential landscape provided by the Mn ions. 
Since $D_{I J}^{-1}(i \omega)$ is real and symmetric, the components of each of its eigenvectors all have the same phase (and can be chosen to be real). Physically this corresponds to the invariance of the system under rotations around the magnetization axis of the collinear state.

The two upper panels of Fig. 19 show results for typical system parameters for two different values of $q_{c}$. Comparison of the panels shows that the effects of the wavevector cutoff on the low-lying excitations have already saturated for the smaller $q_{c}$. Almost all eigenvalues of $D_{I J}^{-1}(0)$ lie at positive energies, but there is often a small fraction of negative eigenvalues, indicating an instability of the collinear state.

In the calculations discussed so far, the Mn positions were chosen completely at random with a uniform distribution, while in a real $(\mathrm{III}, \mathrm{Mn}) \mathrm{V}$ semiconductor the Mn ions are supposed to be located on the cation sites forming an fcc lattice. In the bottom panel of Fig. 19 we show data for the same system parameters as in the top panel but with the Mn positions chosen from an appropriate fcc lattice such that about $5 \%$ of all sites are occupied. Both plots are practically identical indicating that our observations do not depend on this detail of the modeling.

The shape of the eigenvalue distribution of the fluctuation matrix $D_{I J}^{-1}(0)$ is, in the model we have studied, sensitive to the Mn density $N_{M n}$, the carrier density $p$, and the Hamiltonian parameters $m^{*}, J_{p d}$, and $a_{0}$. Situations in which the collinear ferromagnetic state is, for certain disorder realization, stable can be approached most simply, for technical reasons, by letting $a_{0}$ be larger. However for the value of $p / N_{M n}$ illustrated in Fig.19, corresponding to a carrier density somewhat lower than measured in the highest $T_{c}$ samples, negative eigenvalues occur for nearly any Mn ion distribution. We note that negative eigenvalues increase in number as the wavevector cutoff is increased toward its converged value. These results suggest that noncollinear ferromagnetic states are common, that they are sensitive to the distribution of $\mathrm{Mn}$ ions and other defects - especially those that trap carriers, and that the collinear ferromagnetic state tends to become unstable as mean-field band eigenfunctions become more strongly localized around Mn ion site

To further analyze the nature of this instability we consider the participation ratios for these excitations which we define by

$$
p_{j}=\left[N_{M n} V \sum_{I}\left|\alpha_{I}^{j}(E)\right|^{4}\right]^{-1}
$$

where $\alpha_{I}^{j}$ is the $I$-th component of the $j$-th normalized eigenvector of $D_{I J}^{-1}(0)$. The ratio $p_{j}$ is an estimate for the fraction of $\mathrm{Mn}$ sites that have important involvement in the $j$-th spin wave. For example the zero-mode, uniform rotation of all spins, has $p_{j}=1$. Fig.20 shows the disorder-averaged participation ratio as a function of spin-wave energy for the same situation as in Fig.19. The property that negative energy excitations have large participation ratios 


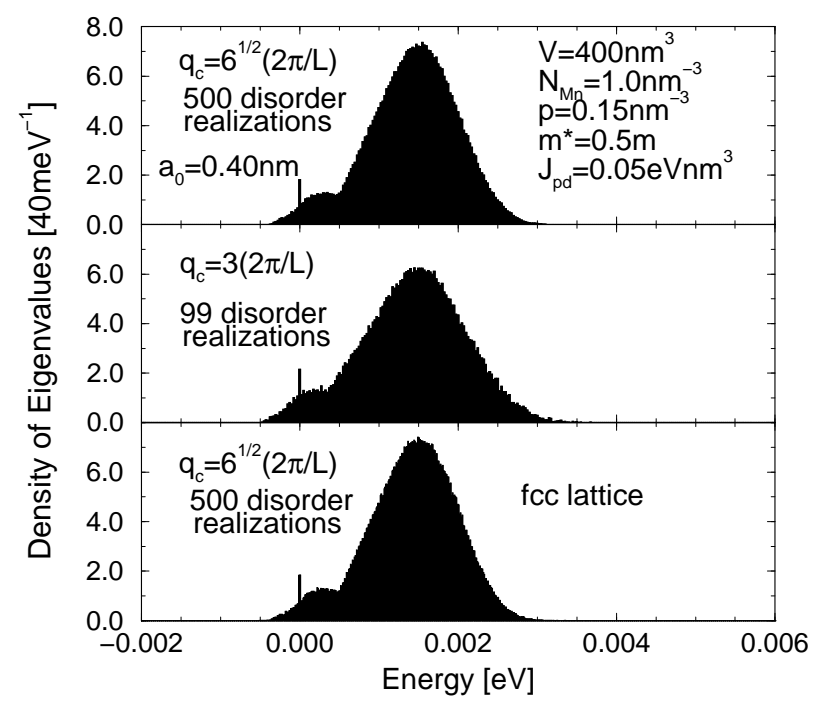

Fig. 19. The disorder-averaged eigenvalue density of the matrix $D_{I J}^{-1}(0)$ describing magnetic fluctuations around the collinear state. The data are obtained for a simulation cube of volume $V=L^{3}=400 \mathrm{~nm}^{3}$ with a Mn density of $N_{\mathrm{Mn}}=1.0 \mathrm{~nm}^{-3}$ and a density of $p=0.15 \mathrm{~nm}^{-3}$ of carriers having a band mass of half the bare electron mass. The strength of the exchange interaction between ions and carriers is $J_{p d}=0.05 \mathrm{eVnm}^{-3}$ with a spatial range of $a_{0}=0.40 \mathrm{~nm}$. The two upper panels show data for different wavevector cutoff $q_{c}$ with the Mn positions chosen completely at random. The lowest panel contains data for the same situation as the top one but with the Mn positions chosen from an fcc lattice. The peaks at zero energy are due to the uniform rotation mode which strictly occurs in any disorder realization.

shows that the instabilities of the collinear state involve correlated reorientations of many spins, rather than lone loosely coupled moments.

Fig. 20 shows the disorder-averaged participation ratio for the same situation as in the top panel of Fig. 19. The negative-energy modes have clearly higher participation ratio than the eigenvectors at positive energy. This shows that the instability of collinear state is due to long-ranged fluctuations involving a large fraction of the spins present in the system. Qualitatively the same observations are made for other values of system parameters.

The effect of a weak external magnetic field on the spin-wave excitation spectrum of the noncollinear state is particularly simple. The field couples to the local moment through its Landé g-factor, adding $2 \mu_{B} H_{e x t}$ to the energy of a spin-wave excitation for $S=5 / 2$, and to the spin and orbital degrees of freedom of the band electrons. The orbital coupling leads to Landau levels that do not play an important role at weak fields in these highly disordered samples. Zeeman coupling is also unimportant, yielding a contribution to the spin-splitting that is negligible compared to the mean-field splitting 


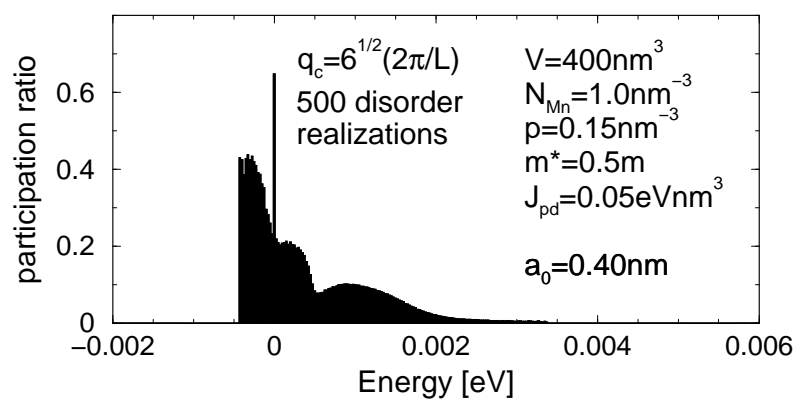

Fig. 20. The disorder-averaged participation ratio for the same situation as in the top panel of Fig. 19. The data is averaged over the sample intervals of the histogram. The value at zero energy is enhanced due to the contribution of the uniform rotation mode in that sample interval.

$\Delta=J_{p d} N_{M n} S \sim 0.1 \mathrm{eV}$. It follows that the most negative eigenvalue of $D_{I J}^{-1}$ is the value of $g_{L} \mu_{B} H_{\text {ext }}$ necessary to force full spin alignment of a noncollinear state. The experiments of Potashnik et al. [52] demonstrate that the maximum value of $M(T=0)$ is achieved over a certain range of annealing histories. We associate this maximum value with the fully aligned collinear Mn configuration state; indeed the maximum moment per $\mathrm{Mn}$ is consistent with full alignment partially compensated by band electrons. For other annealing histories, $M(T=0)$ is reduced, corresponding to noncollinear order of the Mn spins. Calculations like those described above show that these spins gradually align as an external field is added to the Hamiltonian. We expect full alignment to be indicated experimentally by a kink in the $M\left(T=0, H_{\text {ext }}\right)$ curve. At this point, we predict that the system will still have gapless excitations and power-law temperature dependence of the magnetization, in sharp contrast to the gapful excitations and exponentially suppressed temperature dependence that holds for conventional ferromagnets in an external magnetic field. Spin-resonance experiments will nevertheless see a gaped spin-wave spectrum in this regime, since they couple only to the zero mode.

Finally we comment on the degree of spin alignment in the noncollinear state. We generalize our formalism by expanding around and defining HolsteinPrimakoff bosons with respect to a spin coherent state configuration with orientations $\hat{\Omega}_{I}=\left(\sin \vartheta_{I} \cos \varphi_{I}, \sin \vartheta_{I} \sin \varphi_{I}, \cos \vartheta_{I}\right)$. In this general case the effective action has a static contribution (at zero Matsubara frequency only) linear in the Holstein-Primakoff variables:

$$
\mathcal{S}_{\text {fluc }}=\frac{1}{2} \sum_{I}\left[\bar{g}_{I} z_{I}+g_{I} \bar{z}_{I}\right]
$$

with $g_{I}=g_{I}^{1}+i g_{I}^{2}$, and

$$
g_{I}^{1}=\sqrt{2 S}\left(\boldsymbol{e}_{\varphi_{I}} \times \boldsymbol{e}_{z}\right) \cdot \int d^{3} r\left[J\left(\boldsymbol{r}-\boldsymbol{R}_{I}\right)\right.
$$




$$
\begin{aligned}
& \left.\left(\left(\langle\boldsymbol{s}(\boldsymbol{r})\rangle \cdot \boldsymbol{e}_{\varphi_{I}}\right) \boldsymbol{e}_{\varphi_{I}}+\left(\langle\boldsymbol{s}(\boldsymbol{r})\rangle \cdot \boldsymbol{e}_{z}\right) \boldsymbol{e}_{z}\right) \times \boldsymbol{\Omega}_{I}\right] \\
g_{I}^{2}= & \sqrt{2 S} \boldsymbol{e}_{z} \cdot\left(\boldsymbol{e}_{\varphi_{I}} \times \int d^{3} r J\left(\boldsymbol{r}-\boldsymbol{R}_{I}\right)\langle\boldsymbol{s}(\boldsymbol{r})\rangle\right)
\end{aligned}
$$

where $\boldsymbol{e}_{\varphi_{I}}=\left(\cos \varphi_{I}, \sin \varphi_{I}, 0\right)$ and $\boldsymbol{e}_{z}=(0,0,1)$.

The components $g_{I}$ represent the gradient of the energy with respect to distortions parametrized by the $z_{I}$. As above in the case of fluctuations around the collinear state, this zero-frequency contribution can also be obtained via perturbation theory around the given state of Mn spin orientations. The contribution to $\mathcal{S}_{\text {fluc }}$ quadratic in the Holstein-Primakoff variables involves again a proper Matsubara sum and can be obtained straightforwardly. However, its concrete form for the general case is more complicated, and shall not be analyzed here. A given orientation of Mn spins is stationary with respect to fluctuations if all complex coefficients $g_{I}$ vanish. This is the case if and only if $\int d^{3} r J\left(\boldsymbol{r}-\boldsymbol{R}_{I}\right)\langle\boldsymbol{s}(\boldsymbol{r})\rangle$ is collinear with the direction $\hat{\Omega}_{I}$ of the local ion spin. Therefore, the collinear ferromagnetic state is always a stationary (but not necessarily stable) spin state.

We have employed the energy gradient expression (53) in a numerical steepest descent procedure to search for true energy minima. Our results are as follows: In cases where the energy minimum found by this method is close to the collinear state (with a magnetization of about $90 \%$ of the maximum value or more), this minimum appears to be unique for each disorder realization. We can therefore be confident that we have located the absolute ground state of the system. In situations where the magnetization is reduced more substantially, however, (by $\sim 20 \%$ or more) we converge to different energy minima from different starting points. In these cases the model has substantial spin-glass character with a complex energy landscape. This situation occurs typically for larger ratios $p / N_{M n}$. For the system shown in Fig. 20, for instance, magnetization values at local energy minima are typically 30 to $40 \%$ of the collinear state value.

\section{Concluding remarks}

III-V compound semiconductors with Mn substituted for a small fraction of the cations are ferromagnets. In this chapter we have primarily discussed the picture of the physical properties of these materials that follows from a model in which each $\mathrm{Mn}$ produces a $\mathrm{S}=5 / 2$ local moment and acts as an acceptor. The valence band holes and the exchange interaction that couples them to the local moments are both treated phenomenologically. In the simplest approximation the hole bands are taken to be those of the host semiconductor, so that this model has a single unknown parameter, the exchange coupling strength, whose value can be determined experimentally. Many physical properties of this model are relatively simply calculated when a virtual crystal approximation is adopted. When an envelope function approach is used 
to describe the semiconductor bands this is equivalent to replacing the $\mathrm{Mn}$ ions by a continuum, completely eliminating disorder. We have shown that the mean-field theory of this model predicts critical temperatures in good agreement with experiment, that it can describe qualitative features of the magnetic anisotropy energy including the effect of lattice-matching strains between the magnetic epilayer and its substrate, and that it implies values of the anamolous Hall effect often used to characterize these ferromagnets that are in good agreement with experiment. We have also described a theory of the elementary collective spin-wave excitations of the virtual crystal model. By comparing the energy of these elementary excitations with those of the mean-field theory, we are able to judge the reliability of mean-field-theory critical temperature estimates. In this way we find that we reach the wrong conclusion about mean-field theory when we make the apparently innocent choice of using a single parabolic band whose mass is equal to the heavy hole mass of the host semiconductor. For such a model, we find that with typical parameters mean-field theory would give a rather poor estimate of the critical temperature. However when we use a realistic band model with heavy and light holes that are coupled the typical energy of collective magnetic excitations increases substantially. The fact that heavy hole states are given approximately by $\mathrm{J}=3 / 2$ spin coherent states with a $\boldsymbol{k}$ dependent orientation, plays an essential role in producing the enhanced spin stiffness. We conclude that a realistic treatment of the valence band, including its characteristic and strong spin-orbit mixing, is absolutely necessary to achieve even a qualitative understanding of critical temperature trends in these ferromagnets.

Some properties of (III,Mn)V ferromagnets are misrepresented by the virtual crystal approximation. We have examined the effect of Mn site disorder on these ferromagnets both by performing Monte Carlo calculations and by evaluating the collective excitations of disordered systems. The Monte Carlo calculations evaluate the properties of the model exactly for finite systems, except for treating the Mn spin orientations as classical which will have a small effect on thermal magnetization suppression at low temperatures. These calculations confirm conclusions reached by using the virtual crystal approximation for the most part, but also highlight some of its limitations. In particular, while the collinear magnetic state is always stable in the virtual crystal approximation, it can be unstable when disorder is accounted for, leading to non-collinear but still ferromagnetic states. This property provides an explanation to the dependence of a given sample's transition temperature and of the magnetization on details of growth and post-growth annealing procedures.

Research on Mn-doped semiconductors is a very active area of physics, both theoretically and experimentally and there are many topics which we have not been able to even touch upon. For example, studies of nanostructured semiconductor systems with Mn-doped components represent an important component of developments that are aimed towards future spintronic de- 
vices. Nanostructures such as magnetic quantum wells are expected to be unusual ferromagnets because of the possibility of using confinement effects and doping profiles to manipulate their magnetic properties. The transition temperature of these systems can be tuned by external electric field 57, as demonstrated in recent studies of $(\mathrm{In}, \mathrm{Mn})$ As field effect transistors [58. It is also predicted [59] that hysteresis properties of magnetic quantum wells will be extremely sensitive to external bias voltages. As a result, the magnetization orientation in quantum wells can be manipulated electrically without changing the magnetic field. The possibilities for nano-engineering of material properties have already been made apparent by the relatively simple (Ga,Mn)As digital ferromagnetic heterostructures 60]. These systems are grown by incorporating submonolayer planes of MnAs into a GaAs host. They show ferromagnetic transition temperatures up to $50 \mathrm{~K}$ 61.62, the anomalous Hall effect 62] and remarkably square hysteresis loops with higher coercivities [61] than their random alloy counterparts. A confident modeling of these alternative ferromagnetic semiconductors with controlled Mn distribution would significantly contribute to our understanding of magnetic and transport properties of (III,Mn)V ferromagnets.

In this Chapter we have highlighted one particular approach to modeling these materials. We anticipate that other approaches will also bring useful insights. In particular dynamic mean-field-theory calculations [30] are able to capture some of the Mn alloy disorder physics in a relatively simple way, although they do not capture spatial correlations and would not capture non-collinear states. Similarly first principles calculations [18] are likely to prove extremely valuable in the future, once $\mathrm{LDA}+\mathrm{U}$ or dynamical-meanfield theory corrections have been applied to treat the Mn d-electron local moments more accurately.

\section{Acknowledgements}

The work presented in this chapter was done in collaboration with a number of researchers. M. Abolfath, W.A. Atkinson, J. Brum, and Byounghak Lee made important contributions to the mean-field theory of ferromagnetic critical temperature and magnetic anisotropy in DMS's, H.H. Lin has been active in the development of the spin wave theory, Qian Niu's expertise on the semiclassical transport theory has been essential for the anomalous Hall effect study, J. Sinova has been working on correlation effects in the itinerant carrier system, and S.-R.E. Young contributed to the study of disordered DMS's. We thank D.D. Awschalom, D.V. Baxter, R.N. Bhatt, A. Burkov, A. Chattopadhyay, A.I. Chudnovskiy, T. Dietl, J. Fernandez-Rossier, J. Furdyna, E.G. Gwinn, N.A. Hill, A.J. Millis, H. Ohno, F. von Oppen, N. Samarth, D. Sanchez, S. Sanvito, S. Das Sarma, P. Schiffer, M. van Schilfgaarde, and C. Timm for many useful discussions. The work was supported by the Indiana 21st Century Fund, the Welch Foundation, DARPA, DOE, Deutsche 
Forschungsgemeinschaft, the EU COST program, and the Minsitry of Education of the Czech Republic. 


\section{References}

1. H. Ohno, Science 281, 951 (1998); F. Matsukura, H. Ohno, A. Shen, and Y. Sugawara, Phys. Rev. B 57, R2037 (1998).

2. J.M. Kikkawa and D.D. Awschalom, Nature 397, 139 (1999); D.D. Awschalom and N. Samarth, J. Magn. Magn. Mater. 200, 130, (1999); I. Malajovich, J.J. Berry, N. Samarth, and D.D. Awschalom, Nature 411, 770 (2001).

3. J.M. Daughton, J. Appl. Phys. 81, 3758 (1997); J.S. Moodera and G. Mathon, J. Magn. Magn. Mater. 200, 248, (1999); J. Bass and W.P. Pratt, J. Magn. Magn. Mater. 200, 274, (1999); K. O’Grady and H. Laidler, J. Magn. Magn. Mater. 200, 616, (1999).

4. S. Sonoda, S. Shimizu, T. Sasaki, Y. Yamamoto, and H. Hori, condmat/0108159.

5. H. Ohno, J. Magn. Magn. Mater. 200, 110 (1999).

6. Y. Tokura and Y. Tomioka, J. Magn. Magn. Mater. 200, 1, (1999); E. Dagotto, T. Hotta, and A. Moreo, Physics Reports 344, 1 (2001).

7. T. Dietl, H. Ohno, and F. Matsukura, Phys. Rev. B 63, 195205 (2001).

8. F. Matsukura and T. Dietl, to be published.

9. J. Szczytko, A. Twardowski, K. Światek, M. Palczewska, M. Tanaka, T. Hayashi, and K. Ando, Phys. Rev. B 60, 8304 (1999).

10. M. Linnarsson, E. Janzén, B. Monemar, M. Kleverman, and A. Thilderkvist, Phys. Rev. B 55, 6938 (1997).

11. J. Okabayashi, A. Kimura, O. Rader, T. Mizokawa, A. Fujimori, T. Hayashi, and M. Tanaka, Phys. Rev. B 58, R4211 (1998).

12. J. Okabayashi, A. Kimura, T. Mizokawa, A. Fujimori, T. Hayashi, and M. Tanaka, Phys. Rev. B 59, R2486 (1999).

13. J. Okabayashi, A. Kimura, O. Rader, T. Mizokawa, A. Fujimori, T. Hayashi, and M. Tanaka, Phys. Rev. B 64, 125304 (2001).

14. H. Ohno, F. Matsukura, A. Shen, Y. Sugawara, A. Oiwa, A. Endo, S. Katsumoto, and Y. Iye, in Proceedings of the 23rd International Conference on the Physics of Semiconductors, Berlin 1996, edited by M. Scheffler and R. Zimmermann (World Scientific, Singapore, 1996), p. 405.

15. T. Hayashi, H. Shimada, H. Shimizu, and M. Tanaka, J. Cryst. Growth 201/202, 689 (1999).

16. S. Sanvito and N.A. Hill, Phys. Rev. B 62, 15553 (2000); S. Sanvito, P. Ordejón, and N.A. Hill, Phys. Rev. B 63, 165206 (2001); S. Sanvito and N.A. Hill, cond-mat/0108406; M. van Schilfgaarde and O.N. Mryasov, Phys. Rev. B 63, 233205 (2001).

17. H. Akai, Phys. Rev. Lett. 81, 3002 (1998).

18. J.H. Park, S.K. Kwon, and B.I. Min, Physica B 281-283, 703 (2000).

19. J. Kossut, Phys. Stat. Solidi 78, 537 (1976).

20. J.K. Furdyna and J. Kossut, Diluted Magnetic Semiconductors, Vol. 25 of Semiconductor and Semimetals (Academic Press, New York, 1988).

21. B.E. Larson, K.C. Hass, and H. Ehrenreich, Phys. Rev. B 37, 4137 (1988).

22. T. Dietl, Diluted Magnetic Semiconductors, Vol. 3B of Handbook of Semiconductors, (North-Holland, New York, 1994).

23. M. Berciu and R.N. Bhatt, Phys. Rev. Lett. 87, 107203 (2001).

24. A.I. Chudnovskiy, cond-mat/0108396. 
25. W.W. Chow, S.W. Koch, and M. Sargent III, Semiconductor Laser Physics, p.179-192 (Springer-Verlag, Berlin, 1999).

26. M. Abolfath, T. Jungwirth, J. Brum, and A.H. MacDonald, Phys. Rev. B 63, 054418 (2001).

27. T. Omiya, F. Matsukura, T. Dietl, Y. Ohno, T. Sakon, M. Motokawa, and H. Ohno, Physica E 7, 976 (2000).

28. A.K. Bhattacharjee and C. Benoit à la Guillaume, Solid State Comm. 113, 17 (2000).

29. O.M. Fedorych, E.M. Hankiewicz, Z. Wilamowski, and J. Sadowski, condmat/0106227.

30. A. Chattopadhyay, S. Das Sarma, and A.J. Millis, cond-mat/0106455.

31. The role of randomness in a variety of models where spins are coupled to band electrons has been reviewed by A.A. Abrikosov, Advances in Physics 29, 869 (1980).

32. T. Jungwirth, W.A. Atkinson, B.H. Lee, and A.H. MacDonald, Phys. Rev. B 59, 9818 (1999).

33. A. Aharoni, Introduction to the Theory of Ferromagnetism, (Oxford University Press, New York, 1996).

34. I. Vurgaftman, J.R. Meyer, and L.R. Ram-Mohan, J. Appl. Phys. 89, 5815 (2001).

35. T. Dietl, H. Ohno, F. Matsukura, J. Cibert, and D. Ferrand, Science 287, 1019 (2000).

36. T. Jungwirth and A.H. MacDonald, Physica E 10, 153 (2001).

37. T. Jungwirth, J. König, and A.H. MacDonald, to be published.

38. H. Munekata, A. Zaslavsky, P. Fumagalli, and R.J. Gambino, Appl. Phys. Lett. 63, 2929 (1993).

39. R. Skomski and J.M.D. Coey, Permanent Magnetism (Institute of Physics Publishing, Bristol, 1999).

40. T. Jungwirth, Q. Niu, and A.H. MacDonald, cond-mat/0110484.

41. J. Smit, Physica 23, 39 (1958).

42. L. Berger, Phys. Rev. B 2, 4559 (1970).

43. G. Sundaram and Q. Niu, Phys. Rev. B 59, 14915 (1999).

44. J.M. Luttinger, Phys. Rev. 112, 739 (1958).

45. H. Ohno, H. Munekata, T. Penney, S. von Molnár, and L.L. Chang, Phys. Rev. Lett. 68, 2664 (1992).

46. J. König, H.H. Lin, and A.H. MacDonald, Phys. Rev. Lett. 84, 5628 (2000); Physics E 10, 139 (2001).

47. J. König, H.H. Lin, and A.H. MacDonald, in Interacting Electrons in Nanostructures, Eds. R. Haug and H. Schoeller, Lecture Notes in Physics 579, p.195212 (Springer-Verlag, Berlin, 2001).

48. J. Schliemann, J. König, H.H. Lin, and A.H. MacDonald, Appl. Phys. Lett. 78, 1550 (2001).

49. J. Schliemann, J. König, and A.H. MacDonald, Phys. Rev. B 64, 165201 (2001).

50. J. König, T. Jungwirth, and A.H. MacDonald, Phys. Rev. B 64, 184423 (2001).

51. A. Auerbach, Interacting Electrons and Quantum Magnetism (Springer, New York, 1994).

52. S. J. Potashnik, K. C. Ku, S. H. Chun, J. J. Berry, N. Samarth, and P. Schiffer, Appl. Phys. Lett. 79, 1495 (2001). 
53. G. M. Schott, W. Faschinger, and L. W. Molenkamp, cond-mat/0105562.

54. J. Schliemann and A.H. MacDonald, cond-mat/0107573.

55. S. Duane, A.D. Kennedy, B.J. Pendleton, and D. Roweth, Phys. Lett. B 195, 216 (1987).

56. J. König, J. Schliemann, and A.H. MacDonald, unpublished.

57. Byuonghak Lee, T. Jungwirth, and A.H. MacDonald, Phys. Rev. B 61, 15606 (2000).

58. H. Ohno, D. Chiba, F. Matsukura, T. Omiyama, E. Abe, T. Dietl, Y. Ohno, and K. Ohtani, Nature 408, 944 (2000).

59. Byounghak Lee, T. Jungwirth, and A.H. MacDonald, cond-mat/0106536.

60. S.A. Crooker, D.A. Tulchinsky, J. Levy, D.D. Awschalom, R. Garcia, and N. Samarth, Phys. Rev. Lett. 75, 505 (1995).

61. R. K. Kawakami, E. Johnston-Halperin, L.F. Chen, M. Hanson, N. Guébels, J.S. Speck, A.C. Gossard, and D.D. Awschalom, Appl. Phys. Lett. 77, 2379 (2000).

62. H. Luo, B.D. McCombe, M.H. Na, K. Mooney, F. Lehmann, X. Chen, M. Cheon, S.M. Wang, Y. Sasaki, X. Liu, J.K. Furdyna, Proceedings of the 14the International Conference on the Electronic Properties of Two-Dimensional Systems, Prague (2001). 